\title{
Far from obvious: the semantics of locative indefinites
}

\author{
Sela Mador-Haim ${ }^{1}$ Yoad Winter ${ }^{2}$
}

\begin{abstract}
Simple locative sentences show a variety of pseudo-quantificational interpretations. Some locatives give the impression of universal quantification over parts of objects, others involve existential quantification, and yet others cannot be characterized by either of these quantificational terms. This behavior is explained by virtually all semantic theories of locatives. What has not been previously observed is that similar quantificational variability is also exhibited by locative sentences containing indefinites with the 'a' article. This phenomenon is especially problematic for traditional existential treatments of indefinites. We propose a solution where indefinites denote properties and are assigned locations similarly to other spatial descriptions. This Property-Eigenspace Hypothesis accounts for the correlation between the interpretations of locative indefinites and the pseudo-quantificational effects with simple entity-denoting NPs. Thereby, the proposal opens up a new empirical domain for property-based theories of indefinites, with implications for the analysis of collective descriptions, generics, negative polarity items and part-whole structure.
\end{abstract}

Keywords Indefinite $\cdot \mathrm{NP} \cdot$ Locative $\cdot \mathrm{PP} \cdot$ Property $\cdot$ Eigenspace

\section{Introduction}

Existential quantification is traditionally seen as a core element in the meaning of indefinite descriptions. However, applying a simple existential analysis to naturally occurring indefinites has often proven problematic. Indefinites in sentences involving

\footnotetext{
$\bowtie$ Yoad Winter

y.winter@uu.nl

1 University of Pennsylvania, Philadelphia, PA 19104, USA

2 Utrecht University, Utrecht, The Netherlands
} 
generic, predicative, wide-scope and anaphoric effects have all been identified as serious challenges for the traditional existential account. Much work in natural language semantics since the 1970s has been driven by these challenges. This paper addresses yet another puzzle for existential theories of indefinites, which has nonetheless received little attention: the interpretation of indefinites appearing within locative prepositional phrases. The problem was first introduced by Iatridou (2003, 2007), who attributed the observation to Irene Heim (p.c.). The contrast in (1) demonstrates the puzzle.

(1) a. Michael is far from a gas station.

b. Michael is close to a gas station.

In the appropriate context - e.g. a car race in the desert, when Michael's car is running out of gas-sentence (1a) exhibits a universal interpretation: Michael is claimed to be far from all gas stations. By contrast, sentence (1b) has a standard existential interpretation, which only requires there to be one gas station near Michael.

At first blush we may suspect that the contrast in (1) can be explained by postulating some covert negation element within the meaning of far from. This would allow us to derive the universal interpretation of sentence (1a) by assigning the existential denotation of the indefinite narrow scope below the postulated negation. However, the universal interpretation of (1a) only marks one extreme within a variety of non-existential effects with locative indefinites. This is illustrated by the following examples.

(2) a. Michael is more than/less than $5 \mathrm{~km}$ from a gas station.

b. Michael is exactly $5 \mathrm{~km}$ from a gas station.

While the examples in (2a) show a similar contrast to the one between (1a) and (1b), the interpretation of sentence (2b) is more complex. A prominent interpretation of this sentence requires that Michael be exactly $5 \mathrm{~km}$ from the gas station that is nearest to him. This interpretation is not expected by the traditional existential analysis. Furthermore, it cannot be simply described by combining an existential quantifier with covert negation. Similar problems reappear in other cases of indefinites with various locative PPs. We conclude that stipulating negation as part of the meaning of certain locatives would not be a sufficiently general solution to the problem of non-existential locative indefinites.

To solve this problem, we base our analysis on the notion of kinds or properties, which is commonly used in theories of indefinites (Milsark 1974; Carlson 1977; Chierchia 1998). Following these works, and adopting the theoretical setting of Partee (1987), we assume that $a$ indefinites are derivationally ambiguous between properties and existential quantifiers. We propose that property concepts have a spatial dimension similar to entity concepts. This allows us to treat locative indefinites as in (1) and (2) by extending standard analyses of simple locative sentences like Michael is far from/close to London, which make a statement about the distance between two entities. When a region is occupied by a complex entity like London, pseudo-quantificational effects often arise as a direct result of its part-whole structure. Thus, being far from London requires being far from all of its subparts. By contrast, being close to London only requires being close to one of its subparts. Our analysis of the contrast in (1) 
follows from a similar consideration about the location of properties. Sentences (1ab) are treated as making a statement about Michael's distance from the property gas station. The location of a property is assumed to be the union of the regions associated with entities in its extension. With this assumption, sentences $(1 \mathrm{a}-\mathrm{b})$ are analyzed as requiring that Michael be far from/close to the union of all gas station regions. In (1a) this analysis entails that Michael is far from all gas stations. By contrast, in (1b) it only requires that Michael be close to one gas station. Thus, we explain the contrast in $(1 \mathrm{a}-\mathrm{b})$ on the basis of a simple geometrical distinction between the locatives far from and close to, which is observed independently of indefinites. The interpretation of sentences as in (2), as well as other non-existential effects with locative indefinites, are similarly explained by geometrical considerations about the meaning of spatial concepts. The pseudo-quantificational effects with locative indefinites constitute a new type of evidence for property-based treatments of indefinites that have been proposed in the last two decades (Zimmermann 1993; Van Geenhoven 1996; McNally 1998, 2011, 2014; Chung and Ladusaw 2003; Van Geenhoven and McNally 2005; among others). Furthermore, as we will show, the property-based analysis of locative indefinites also has broader implications for the analysis of collective descriptions, negative polarity items and part-whole structure.

Section 2 introduces standard assumptions on indefinites and locatives, together with our main hypothesis about how properties are assigned a location, or eigenspace. In Sect. 3 we show that this analysis deals successfully with a variety of locative sentences with indefinites. Section 4 gives further support for this analysis by showing its advantages over putative accounts using covert negation. Section 5 discusses some broader implications of the proposed account as well as some open problems.

\section{The basic proposal}

The key elements of our proposal are the property denotation of $a$ indefinites and a general semantics of locatives. We start out by reviewing two approaches to indefinites in the literature: the quantificational approach and the predicative, or property-based approach. Following Partee (1987) and others, we assume that $a$ indefinites in English are initially property-denoting, but they may end up denoting existential quantifiers due to a derivational mechanism. We show how this approach leads to systematic ambiguity with locative indefinites. To analyze this ambiguity, we review some basic notions from previous work on locative expressions. Then we introduce our main contribution to the analysis of this puzzle: the assumption that properties can be located like ordinary entities, and the principle governing this procedure, which we call the Property-Eigenspace Hypothesis.

\subsection{Indefinites and properties}

Since Montague (1973), many works on natural language semantics have analyzed all NPs as denoting generalized quantifiers. Notably, Barwise and Cooper (1981) treat items like $a$ and some as denoting existential determiners. This classic approach analyzes all occurrences of indefinites as existential or 'intersective' generalized quan- 
tifiers (Keenan and Westerståhl 2011). An alternative view in semantic theory has been to analyze at least some indefinites as denoting properties (Milsark 1974). ${ }^{1}$ Under this analysis the indefinite article $a$ has a null contribution to the NP's denotation. Accordingly, the basic denotation of an indefinite like a gas station is assumed to be the same property denoted by the nominal part gas station. This approach directly derives a proper interpretation for sentences with predicative indefinites, e.g. this is a gas station. By contrast, with indefinites in argument positions (e.g. I see a gas station), the existential or 'specific' interpretation has to be derived by an additional semantic process. In such argument positions, Partee (1987) assumes an existential operator which applies to properties as part of the interpretation of the indefinite. Thus, in Partee's account indefinites are derivationally ambiguous between properties and existential quantifiers.

These standard procedures are also applicable to indefinites that appear within prepositional phrases. Standardly, PPs like in NYC and above NYC are analyzed as determining positions with respect to the 'landmark' object, in this case New York City. Such PPs that specify relations with respect to a landmark are referred to as locative PPs, or simply locatives. Locative PPs are distinguished from directional PPs like through NYC and from NYC that determine paths or motions relative to the landmark. When a locative like in a city contains an indefinite NP, we refer to that indefinite as a locative indefinite. Following Partee's flexible account, we embrace two derivations for sentences with locative indefinites. For instance, sentence (3) below (=(1a)) is analyzed as derivationally ambiguous between the two representations sketched in $(3 \mathrm{a}-\mathrm{b})$.

(3) Michael is far from a gas station.

a. Michael is [[far from] [E [a gas station]]]

b. Michael is [[far from] [a gas station]]

Under the analysis in (3a), a covert existential process applies within the indefinite NP before it composes with the preposition. In analysis (3b), the preposition's denotation directly combines with the property denotation of the indefinite. We use the notation GS for the property denoted by the indefinite a gas station, and gs for the set associated with the extension of this property. Accordingly, (3a) leads to the standard analysis in (4a) below. By contrast, in (3b) the property GS remains intact and serves as the direct argument of the locative, as formalized in (4b).

(4) a. $\exists x \in$ gs. far_from $(\mathbf{m}, x)$

b. far_from $(\mathbf{m}, G S)$

The choice between these two readings is affected by pragmatic factors, most of them beyond the scope of this paper. What is crucial for our purposes is that both strategies are attested with $a$ indefinites in one context or another. For instance, as we

\footnotetext{
${ }^{1}$ For more recent works following Milsark's approach see Zimmermann (1993), Van Geenhoven (1996, 1998), McNally (1998, 2009, 2011, 2014), Dayal (1999), Condoravdi et al. (2001), Chung and Ladusaw (2003), Van Geenhoven and McNally (2005), among others. Other theories often use properties, or kinds, for treating generic indefinites (Carlson 1977; Carlson and Pelletier 1995; Chierchia 1998). Throughout this paper we use the term properties since the difference between them and kinds is not crucial for our purposes.
} 
will see, for sentence (3), the property-based analysis (4b) gives a correct interpretation, whereas the standard existential analysis (4a) does not. This existential analysis becomes more useful when we consider indefinites with more descriptive content such as a gas station I know. When used in locatives, such indefinites predominantly show an existential/specific interpretation. Similar interpretations are also salient with the indefinites in (5) below.

(5) Michael is far from somela certain gas station.

In contrast with $a$ indefinites, the indefinites in (5) are unambiguously existential or specific. We capture this interpretation by assuming that such indefinites only have the existential analysis in (4a). ${ }^{2}$ We should note that although for simplicity we adopt Partee's existential analysis, this does not do justice to specificity and other notorious phenomena in the semantics and pragmatics of indefinites. ${ }^{3}$ To highlight this shortcoming, we informally describe existential/specific interpretations of locative indefinites as in (6) below.

(6) Michael is far from $\operatorname{somE}_{\exists}$ gas station.

This notation can be read as having the same import as (4a), but it is also used in order to convey our conviction that the $a$ indefinite in (3) is equivalent in one of its readings to the some indefinite in (5), while avoiding details about this existential/specific analysis. Further, NPs that are unambiguously quantificational can also appear in locative sentences, e.g. Michael is far from every gas station. Such locative sentences do not require any predication over properties. Rather, they are analyzed as quantification over entities and treated by standard assumptions about quantifier scope (Barker 2015).

\subsection{Locatives and eigenspaces}

As we saw, sentence (3) has a prominent interpretation that is not derived by simple assumptions on existential quantification or specificity. We informally paraphrase this interpretation by writing 'Michael is far from EVERY $_{\forall}$ gas station'. However, since we do not assume that this interpretation involves a universal quantifier over entities, we refer to it as pseudo-universal. Analysis (4b) models the pseudo-universal interpretation by letting the locative relation apply to the entity denotation of the name Michael and the property denotation of the indefinite a gas station. Our challenge is to explain how properties stand in such locative relations to entities, examine the interpretations that this option derives and check whether they conform with linguistic intuitions.

We start out by reviewing some standard assumptions on the semantics of locative PPs (Wunderlich 1991; Zwarts and Winter 2000). Let us first consider the simple locative sentence (7) below.

\footnotetext{
2 McNally's (1998) property-based account of there sentences might require that the indefinites in (5) also denote properties, and not only existential quantifiers. See more on this point in Sect. 5.

3 See Fodor and Sag (1982), Reinhart (1997), Winter (2001), Von Heusinger (2011) on widescope/specificity; Kamp (1981), Heim (1982), Groenendijk and Stokhof (1991), Kamp and Reyle (1993) on discourse binding.
} 
(7) Michael is far from London.

At the compositional level, sentence (7) expresses a binary relation between entities. This is formalized below.

\section{(8) far_from $(\mathbf{m}, \mathbf{l})$}

In this analysis, the entity $\mathbf{l}$ is the landmark and the entity $\mathbf{m}$ is the located object. More explicitly, the analysis in (8) is expanded to the following spatial analysis.

(9) FAR_FROM $(\operatorname{LOC}(\mathbf{m}), \operatorname{LOC}(\mathbf{l}))$

In (9), the relation FAR_FROM is the two-place locative predicate underlying the meaning of the binary relation far_from. The relation FAR_FROM holds in (9) between two spatial objects: the locations $\operatorname{LOC}(\mathbf{m})$ and $\operatorname{LOC}(\mathbf{l})$ of the entities $\mathbf{m}$ and $\mathbf{l}$. Following Wunderlich (1991), we refer to the location of an entity as the entity's eigenspace. ${ }^{4}$ As we formally elaborate below, we use points and regions (=sets of points) as the spatial semantic objects that model eigenspaces. In locative sentences, we assume that the eigenspace of the located object is a point, whereas the landmark has a region as its eigenspace (see fn. 5 below). When the eigenspace of the entity for Michael is the point $m$, and the eigenspace of London is the region $L$, we write:

(10) $\operatorname{LOC}(\mathbf{m})=m ; \quad \operatorname{LOC}(\mathbf{l})=L$

Summing up, we treat the logical form (8) as expressing a spatial relation FAR_FROM between the point $m$ and the region $L$. In formula:

\section{(11) FAR_FROM $(m, L)$}

This analysis immediately expects a pseudo-universal interpretation of sentence (7). By basic properties of the locative relation FAR_FROM, which we spell out formally in the next section, it follows that when a point $m$ is far from a region $L$, it is far from every point in $L$. Thus, analysis (11) expects that sentence (7) is interpreted similarly to the statement 'Michael is far from EVERY $\forall$ part of London'. This observation on entity-denoting noun phrases is unsurprising (Iris et al. 1988; Casati and Varzi 1999; Johansson 2004). However, when it is combined with the property analysis of indefinites, it provides a straightforward account of their puzzling behavior in locative sentences.

\subsection{Locative indefinites: the Property-Eigenspace Hypothesis}

Our account of the pseudo-universal interpretation of (3) follows from the analysis in (4b), where the spatial relation holds between an entity and a property. To deal with this situation, we let any spatial property have an eigenspace of its own, similar to spatial entities. Thus, when locating the entity $\mathbf{m}$ and the property GS we get the following spatial-level analysis of (4b).

\footnotetext{
${ }^{4}$ German eigen $=$ 'its own'. Our intuitive use of the term eigenspace should not be confused with the mathematical notion of eigenspace in Linear Algebra.
} 
(12) FAR_FROM $(\operatorname{LOC}(\mathbf{m}), \operatorname{LOC}(G S))$

Here the spatial relation FAR_FROM holds between Michael's eigenspace and the eigenspace for the property GS. The main new element of our proposal is in the way properties are located. We define the eigenspace of properties on the basis of the eigenspaces of entities in their extension. Specifically, we adopt the following hypothesis.

(13) Property-Eigenspace Hypothesis ( $\mathrm{PEH):} \mathrm{A} \mathrm{property's} \mathrm{eigenspace} \mathrm{is} \mathrm{the}$ union of eigenspaces for entities in its extension.

In our analysis (12) of sentence (3), the PEH requires that the eigenspace LOC(GS) of the property $G S$ be the union of eigenspaces for entities in the set gs, the extension of the property. In formula:

(14) $\operatorname{LOC}(G S)=\bigcup\{\operatorname{LOC}(x): x \in \mathbf{g s}\}$

Summing up, we get the following property-based analysis of sentence (3):

(15) FAR_FROM $(m, \bigcup\{\operatorname{LOC}(x): x \in \mathbf{g s}\})$

In words, (15) states that the point $m$ for Michael's location is far from the region composed of all gas station regions. As we show in detail in Sect. 3, this statement correctly captures the pseudo-universal interpretation of the locative indefinite in (3). More generally, our analysis derives non-existential interpretations of locative indefinites by using the same principles that account for locative sentences with referential NPs. Because of this reduction, the PEH expects the following generalization.

(16) PEH Generalization: The pseudo-quantificational behavior of locative indefinites correlates with the pseudo-quantificational behavior of locative referential NPs.

This generalization makes a connection between the interpretation of locative indefinites, and the geometrical aspects of locative meanings as attested with pseudoquantificational interpretations of locative referential NPs. It should be kept in mind that we assume that, in addition to their property-based analysis, all locative indefinites also have standard existential/specific readings. The correlation expected in (16) only concerns pseudo-quantificational interpretations of locative sentences with indefinites, and not their standard existential reading. For instance, as we have seen, the pseudouniversal interpretation of the indefinite a gas station in sentence (3) correlates with a pseudo-universal interpretation of the proper name London in (7). According to the PEH generalization, this type of correlation should hold for all pseudo-quantificational interpretations of locative indefinites. Specifically, for any non-existential interpretation of locative indefinites, the PEH generalization expects to find a parallel effect with locative referential NPs, and vice versa. Section 3 shows empirical support for this expectation.

\section{Evidence for the Property-Eigenspace Hypothesis}

This section lays out the main arguments for our property-based approach and the PEH. We go over different types of locative relations, the non-existential effects they lead 
to with indefinites, and the way these effects are accounted for in our proposal. This account supports the PEH generalization, hence the PEH itself, and more generally, the property-based account of $a$ indefinites.

\subsection{A technical preliminary: topological spaces}

In Sect. 2 and throughout this paper, we treat eigenspaces of landmarks (e.g. London in (7)) as regions. Eigenspaces of located objects (e.g. Michael in (7)) are unrealistically modeled as points. Accordingly, locative relations are treated as relations between points and regions. ${ }^{5}$ We model points as elements of a topological space, where regions are sets of such points. Standardly, a topological space is a structure $\langle M, T\rangle$ where $M$ is a non-empty set and $T$ is a collection of subsets of $M$. The collection $T$ includes the empty set and $M$ itself. Furthermore, $T$ is closed under arbitrary unions and finite intersections. ${ }^{6}$ Topological spaces are formally defined below, with the accompanying standard notions of open and closed sets.

Definition 1 (topological space, open/closed sets) Let $M$ be a non-empty set and let $T \subseteq \wp(M)$ be a collection of subsets of $M$, where $\varnothing \in T, M \in T$, and for every $\mathcal{A} \subseteq T$ : $\cup \mathcal{A} \in T$, and furthermore, $\cap \mathcal{A} \in T$ if $\mathcal{A}$ has finitely many elements. The pair $\langle M, T\rangle$ is a topological space, the sets in $T$ are called open and their complements in $M$ are called closed.

Henceforth we avoid referring to the set $T$ of open sets, and sloppily refer to the set $M$ itself as a 'topological space'. The elements of $M$ are what we intuitively refer to as 'points', whereas 'regions' are subsets of $M$.

\subsection{Topological locatives}

Topological locative concepts are those geometrical concepts that can be defined by only referring to a topological space (i.e. points and regions), without invoking further geometrical concepts like distances or directions. The basic topological concepts we treat are INSIDE and OUTSIDE. Some English locatives that express these concepts are given below.

$$
\begin{aligned}
& \text { Topological locatives } \\
& \text { INSIDE: in, inside (of), within } \\
& \text { OUTSIDE: out of, outside (of), without }
\end{aligned}
$$

Concentrating on the topological usage of the prepositions in (17), let us consider the antonyms inside and outside in the following simple sentences.

\footnotetext{
5 A more general treatment would model locatives as binary relations between regions. However, that would introduce complications that are unnecessary for our purposes here. See Zwarts and Winter (2000) for an analysis where all eigenspaces are uniformly treated as regions.

${ }^{6}$ I.e. for any subset $\mathcal{A}$ of $T$, the union $\cup \mathcal{A}$ is in $T$, and if $\mathcal{A}$ has finitely many members then the intersection $\cap \mathcal{A}$ is also in $T$ (see Definition 1).
} 
(18) a. The visitor is inside the building.

b. The visitor is outside the building.

Sentence (18a) has the pseudo-existential interpretation paraphrased in (19a) below, whereas (18b) has the pseudo-universal interpretation paraphrased in (19b).

(19) a. The visitor is inside $\operatorname{SOME}_{\exists}$ part of the building.

b. The visitor is outside EVERY $\forall$ part of the building.

In correspondence with this pseudo-quantificational alternation in (18) we also observe an existential/universal contrast with indefinites appearing with INSIDE and OUTSIDE locatives. To illustrate that, let us consider the following sentences collected from the internet.

(20) a. Every vehicle or trailer which is parked outside of a garage shall display license plates with current registration tabs.

b. I personally find the planets that formed outside of a star system more fascinating than ejecta.

c. One-third of the funded proposals shall serve schools within a Metropolitan County, and at least one-third shall serve schools outside of a Metropolitan County.

In (20a), the vehicles and trailers quantified over are those that are outside of any garage. Similarly, in (20b), the planets referred to are those that are formed outside of any star system. Thus, the indefinites within the outside of locatives of (20a) and (20b) are interpreted universally. In sentence (20c), the interpretation of the indefinite with the preposition outside of is again universal. However, the indefinite with the preposition within is existential. Thus, the schools that are served include those that are within some metropolitan county and those that are outside of all the relevant metropolitan counties.

Although we assume a topological space as the basis for our spatial model, for the topological concepts INSIDE and OUTSIDE we do not exploit the full power of this ontology. We define these locative relations by merely appealing to set-membership between points and regions. Below we define these two concepts.

(21) For all points $x$ and regions $A$ :

$$
\begin{aligned}
& \operatorname{Inside}(x, A) \Leftrightarrow x \in A \\
& \operatorname{Outside}(x, A) \Leftrightarrow x \notin A
\end{aligned}
$$

In words: a point $x$ is inside a region $A$ if $x$ is a member of $A ; x$ is outside $A$ if $x$ is not in $A$. This immediately leads to the following observations.

$$
\begin{array}{ll}
\text { a. } & \operatorname{Inside}(x, A) \Leftrightarrow \exists y \in A \cdot \operatorname{inside}(x,\{y\}) \\
\text { b. } & \operatorname{Outside}(x, A) \Leftrightarrow \forall y \in A \cdot \operatorname{Outside}(x,\{y\})
\end{array}
$$

In words: a point $x$ is inside a region $A$ iff $x$ is inside some singleton sub-region $\{y\}$ of $A ; x$ is outside $A$ iff $x$ is outside every such singleton.

Having observed these facts, we cannot be surprised by the equivalences between the locative sentences $(18 \mathrm{a}-\mathrm{b})$ and the quantificational statements in (19a-b). For 
instance, consider the equivalence $(18 b) \Leftrightarrow(19 b)$. When sentence (18b) holds, by (22b) we conclude that any part of the building must consist of points that are disjoint from the eigenspace of the visitor, hence the visitor is outside every such part, i.e. (19b) holds. Conversely, when (19b) holds, observation (22b) means that the visitor's eigenspace is different than any point within any part of the building. Therefore, the visitor's eigenspace is outside the building, as (18b) states. The equivalence $(18 a) \Leftrightarrow(19 a)$ is similarly accounted for by observation (22a). In more general terms, this analysis explains why OUTSIDE locatives support pseudo-universal interpretations with respect to the part-whole structure of the landmark, while INSIDE locatives support pseudoexistential interpretations.

Now let us go back to locative indefinites. In (20) we demonstrated that indefinites in topological PPs may show a similar pattern to locative referential NPs: pseudoexistential interpretations with INSIDE locatives and pseudo-universal interpretations with OUTSIDE locatives. The PEH derives such pseudo-quantificational effects with indefinites as stemming from the same considerations that we used to account for sentences (18a-b). Let us exemplify that using the following simplified versions of the sentences in (20c).

(23) a. The school is within a metropolitan county.

b. The school is outside of a metropolitan county.

First, because we use Partee's assumption on the flexibility of $a$ indefinites, both sentences in (23) are expected to have an existential reading. However, in addition to this standard analysis, we also expect a property-based interpretation, derived by the PEH. To see what this interpretation amounts to, let us use $M C$ for the property denotation of metropolitan county, where mc is the set of entities in its extension. Recall that the PEH takes the eigenspace of the property $M_{C}$ to be the union of eigenspaces of entities in mc. This means that the eigenspace $\operatorname{LOC}(M C)$ is treated as the union $\cup \mathcal{A}$, where $\mathcal{A}$ is the collection of eigenspaces $\{\operatorname{LOC}(x): x \in \mathbf{m c}\}$. As a direct result from our observation in (22), we conclude that for every collection of regions $\mathcal{A}$, for every point $x$ :

$$
\begin{array}{ll}
\text { a. } & \operatorname{Inside}(x, \cup \mathcal{A}) \Leftrightarrow \exists A \in \mathcal{A} \cdot \operatorname{Inside}(x, A) \\
\text { b. } & \operatorname{Outside}(x, \cup \mathcal{A}) \Leftrightarrow \forall A \in \mathcal{A} \cdot \operatorname{dutside}(x, A)
\end{array}
$$

In words: a point $x$ is inside the union of the regions in $\mathcal{A}$ iff $x$ is inside some region $A$ in $\mathcal{A} ; x$ is outside this union iff $x$ is outside every region in $\mathcal{A}$.

This observation explains the behavior of the locative indefinites in (23) as following from the PEH. In (25a-b) we see this more concretely by considering our PEH-based analyses of sentences (23a-b), where the point $s$ is the school's location.

$$
\begin{array}{rll}
\text { a. } & \operatorname{InSide}(s, \operatorname{LOC}(M C)) & \triangleright \text { analysis of (23a) } \\
& \Leftrightarrow \operatorname{Inside}(s, \bigcup\{\operatorname{LOC}(x): x \in \mathbf{m c}\}) & \triangleright \text { by PEH } \\
& \Leftrightarrow \exists x \in \mathbf{m c} \cdot \operatorname{Inside}(s, \operatorname{LOC}(x)) & \triangleright \text { by }(24 \mathrm{a}) \\
\text { b. } & \operatorname{OUtside}(s, \operatorname{LOC}(M C)) & \triangleright \text { analysis of }(23 \mathrm{~b}) \\
& \Leftrightarrow \operatorname{Outside}(s, \bigcup\{\operatorname{LOC}(x): x \in \mathbf{m c}\}) & \triangleright \text { by PEH } \\
& \Leftrightarrow \forall x \in \mathbf{m c} \cdot \operatorname{Outside}(s, \operatorname{LOC}(x)) & \triangleright \text { by }(24 \mathrm{~b})
\end{array}
$$


The analysis in (25a) means that the only pseudo-quantificational effect that the PEH expects for $a$ indefinites with INSIDE locatives is existential. Thus, in this case the property-based analysis converges with the standard quantificational analysis. By contrast, the analysis in (25b) means that the PEH expects OuTSIDE locatives to give rise to pseudo-universal effects with $a$ indefinites, in addition to their standard existential reading. This distinction between INSIDE indefinites and OUTSIDE indefinites accounts for the contrast in (20c) above.

\subsection{Distal locatives and 'outside' presuppositions}

Locatives like far from and close to introduce requirements on the distance between eigenspaces. English distal locatives often involve a predicate over distances (far, close, 20km) and a directional preposition (from, to). We distinguish between distal locatives according to the monotonicity of their distance predicate. Intuitively, upward/downward monotonicity of the distance predicate is characterized as its closure under lengthening/shortening of distances within it. For instance, we say that the adjective far is an upward-monotone distance predicate, because if a distance is considered far, then any bigger distance is considered far as well. Accordingly, we classify the locative far from as an upward-monotone distal locative (DISTM $\uparrow$ ). Analogously, the locative close to is classified as downward monotone (DISTM $\downarrow$ ). Now let us consider the distance predicate exactly $20 \mathrm{~km}$. A distance of exactly $20 \mathrm{~km}$ is within this predicate, but if we lengthen or shorten such a distance, we may get out of the predicate's extension. Because of that, we classify the distal expression exactly $20 \mathrm{~km}$ from as a non-monotone locative ( DIST $_{\mathrm{M}_{\neg}}$ ). Some more examples for the three types of distal locatives are given below.

\section{Distal locatives}

$$
\begin{array}{ll}
\operatorname{DIST}_{\mathrm{M}} \uparrow & \text { far from, away from, more than/at least } 20 \mathrm{~km} \text { (away) from } \\
\operatorname{DIST}_{\downarrow}: & \text { close to, near (to), less than/at most } 20 \mathrm{~km} \text { (away) from } \\
\operatorname{DIST}_{\neg}: & \text { exactly } 20 \mathrm{~km} \text { (away) from, between } 20 \mathrm{~km} \text { and } 30 \mathrm{~km} \text { (away) from }
\end{array}
$$

The monotonicity properties of distal locatives affect pseudo-quantificational effects in simple locative sentences. For the monotone distal locatives at least $/$ most $20 \mathrm{~km}$ from, this is illustrated by the intuitive equivalences between sentences $(27 \mathrm{a}-\mathrm{b})$ below and the respective sentences in $(28 \mathrm{a}-\mathrm{b})$.

(27) a. Michael is at least $20 \mathrm{~km}$ from London.

b. Michael is at most $20 \mathrm{~km}$ from London.

(28) a. Michael is at least $20 \mathrm{~km}$ from EVERY $_{\forall}$ part of London.

b. Michael is at most $20 \mathrm{~km}$ from $\operatorname{some}_{\exists}$ part of London.

The interpretation of (27a) is pseudo-universal similarly to the behavior of the DISTM $\uparrow$ locative far from (cf. (7)). Sentence (27b) is only interpreted as pseudo-existential, similarly to the behavior of the parallel sentence with the $\operatorname{DIST}_{\downarrow} \downarrow$ locative close to. 
When monotone distal locatives combine with indefinites, we observe pseudoquantificational variability similar to (27). We illustrate that using the intuitive interpretations of sentences $(29 a-b)$ below as paraphrased in $(30 a-b)$, respectively.

(29) a. Michael is at least $20 \mathrm{~km}$ from a gas station.

b. Michael is at most $20 \mathrm{~km}$ from a gas station.

a. Michael is at least $20 \mathrm{~km}$ from EverY $\forall$ gas station.

b. Michael is at most $20 \mathrm{~km}$ from $\operatorname{sOME}_{\exists}$ gas station.

In (29a) the $\operatorname{DIST}_{\mathrm{M} \uparrow}$ locative leads to a pseudo-universal interpretation, parallel to the pseudo-universal interpretation of (27a) above. This is the same parallelism we observed with the DIST $\mathrm{M}_{\uparrow}$ locative far from in (3) and (7). In (29b) the DIST $\mathrm{M}_{\downarrow}$ locative supports a (pseudo) existential interpretation parallel to the interpretation of (27b) above. These parallelisms between (29) and (27) show further support for our PEH generalization in (16). To see how the PEH accounts for these data, let us look further into the formal semantics of distal locatives. We endow the spatial ontology with a metric function $d$ that models distances between points as real numbers. Formally, a metric function over a set $M$ is defined as follows.

Definition 2 (metric function) Let $M$ be a non-empty set, and let $d$ be a function from the cartesian product $M \times M$ to non-negative real numbers in $\mathcal{R}$. The function $d$ is called a metric function if it satisfies the following requirements for all elements $x, y \in M$ :

$$
\begin{aligned}
& d(x, y)=d(y, x) \quad \text { (symmetry) } \\
& d(x, y)+d(y, z) \geq d(x, z) \quad \text { (triangle inequality) } \\
& d(x, y)=0 \text { iff } x=y \quad \text { (identity of indiscernibles) }
\end{aligned}
$$

The set $M$ together with the metric function $d$ are called a metric space. Any metric space can be naturally defined as a topological space (Kelley 1955, p. 119). We let a metric space $M$ with its natural topology be the spatial ontology for distal locatives. Definition 3 below naturally extends the metric function $d$ to measure distances between points and closed regions. ${ }^{7}$

Definition 3 (distance) For every non-empty closed region $A \subseteq M$ and a point $x \in M$ not in $A$, the distance between $x$ and $A$ is defined by:

$$
\mathbf{d}(x, A)=\min (\{d(x, y): y \in A\}) \text {. }
$$

In words: $\mathbf{d}(x, A)$ is the minimal distance between $x$ and points in $A$. Here and henceforth, we assume for simplicity that all eigenspace regions are closed. Further, Definition 3 ignores cases where the point $x$ is in the region $A$. The reason for this will be clarified as we go along.

A distal locative concept is defined on the basis of its distance predicate, or the corresponding set of distances. Suppose that $R$ is a set of non-negative real numbers that correspond to distances in a metric space. On the basis of the set $R$, a distal relation $\operatorname{DIST}_{R}$ is defined as follows.

\footnotetext{
7 On closed regions see Definition 1 above. Using closed regions allows us to analyze a distance from a region as a minimum rather than an infimum. On the justification for the assumption in Definition 3 that the point $x$ is not in $A$, see our discussion below of 'outside presuppositions'.
} 
(31) For any region $A$ and point $x \notin A$ :

$$
\operatorname{DisT}_{R}(x, A) \Leftrightarrow \mathbf{d}(x, A) \in R
$$

In words: for any set $R$ of non-negative real numbers, the relation $\operatorname{DIST}_{R}$ holds between a point $x$ and a region $A$ if the distance between $x$ and $A$ is a number in $R$.

Specific distal locatives are directly derived by the definition in (31) with various distance predicates determining the set $R$. For the locative far from, we assume that the distal predicate far contributes as the set $R$ a semi-open interval $(r, \infty)$, of all the real numbers bigger than some contextually given $r$. By using this interval, (31) derives a definition for the locative relation FAR_FROM. Formally, for every region $A$ and point $x \notin A$ we have:

$$
\underset{\operatorname{FAR} \_ \text {FROM }}{ }(x, A) \Leftrightarrow \operatorname{DiST}_{(r, \infty)}(x, A) \Leftrightarrow \mathbf{d}(x, A)>r
$$

In words: a point $x$ is considered far from a region $A$ iff the distance between $x$ and $A$ is bigger than $r$. We note that the interval $(r, \infty)$ corresponding to the predicate far is upward-monotone in the following sense: for every real number $r^{\prime}$ in $(r, \infty)$, any number bigger than $r^{\prime}$ is in $(r, \infty)$ as well. As we will see below, central semantic properties of the locative far from follow directly from this upward-monotonicity of the interval $(r, \infty)$.

Other distal concepts are similarly derived from (31), by specifying the real numbers in the set $R$ of distances in the extension of the distance predicate. Thus, semantic properties of distal locatives are systematically affected by monotonicity properties of the distance predicate that they contain. For the DIST $\mathrm{M} \uparrow$ locative at least $20 \mathrm{~km}$ from we assume that the set $R$ is the interval $[20, \infty)$ of real numbers that model the distances of $20 \mathrm{~km}$ and more. Accordingly we denote:

$$
\text { AT_LEAST_20KM_FROM }(x, A) \Leftrightarrow \operatorname{DIST}_{[20, \infty)}(x, A) \Leftrightarrow \mathbf{d}(x, A) \geq 20
$$

In words: the point $x$ is at least $20 \mathrm{~km}$ from the region $A$ iff the distance between them is greater or equal than 20 . The interval $[20, \infty)$ in (33) is again upward-monotone.

When the set $R$ of real numbers is upward-monotone as in (32) and (33), we get the following fact, similar to fact (24b) about OUTSIDE locatives.

(34) Let $R$ be an upward-monotone set of distances. For any collection of regions $\mathcal{A}$ and a point $x \notin \cup \mathcal{A}$, we have:

$$
\operatorname{DisT}_{R}(x, \cup \mathcal{A}) \Leftrightarrow \forall A \in \mathcal{A} \cdot \operatorname{DisT}_{R}(x, A)
$$

In words: when $R$ is upward-monotone, the distance between a point $x$ and the union of regions in $\mathcal{A}$ is in $R$ iff the distance between $x$ and every region $A$ in $\mathcal{A}$ is in $R$. Specifically in our examples for DISTM $\uparrow$ locatives, we get:

$$
\begin{aligned}
& \text { a. } \operatorname{FAR} \_ \text {FROM }(x, \cup \mathcal{A}) \quad \Leftrightarrow \forall A \in \mathcal{A} \text {.FAR_FROM }(x, A) \\
& \text { b. AT_LEAST_20KM_FROM }(x, \cup \mathcal{A}) \Leftrightarrow \forall A \in \mathcal{A} \text {.AT_LEAST_20KM_FROM }(x, A)
\end{aligned}
$$

These equivalences immediately account for the pseudo-universal interpretations of sentences with DIST $\mathrm{M} \uparrow$ locatives. First, sentence (7) ('Michael is far from London') is analyzed as equivalent to Michael is far from EVERY $_{\forall}$ part of London, and similarly for 
the equivalence $(27 \mathrm{a}) \Leftrightarrow(28 \mathrm{a})$ with the predicate at least $20 \mathrm{~km}$. We employ a similar analysis for the pseudo-quantificational interpretation of $a$ indefinites with DISTM $\uparrow$ locatives. Let us consider for instance the PEH-based analysis below of sentence (29a) (='Michael is at least $20 \mathrm{~km}$ from a gas station'), and the equivalence it gives rise to. ${ }^{8}$

$$
\begin{array}{ll}
\text { AT_LEAST_20KM_FROM }(m, \operatorname{LOC}(G S)) & \triangleright \text { analysis of (29a) } \\
\Leftrightarrow \text { AT_LEAST_20KM_FROM }(m, \bigcup\{\operatorname{LOC}(x): x \in \mathbf{g s}\}) & \triangleright \text { by PEH } \\
\Leftrightarrow \forall x \in \text { gs.AT_LEAST_20KM_FROM }(m, \text { LOC }(x)) & \triangleright \text { by }(35 \mathrm{~b})
\end{array}
$$

This is the pseudo-universal interpretation of (29a), typical of many DIST $\mathrm{M} \uparrow$ indefinites.

We would now like to consider the formal treatment of DIST ${ }_{\downarrow}$ locatives like close to. However, before moving on there is a basic empirical question about such locatives that we need to address: can we indeed classify their pseudo-quantificational behavior as existential? Let us consider example (37a) below, and the attempt to paraphrase it in $(37 b)$.

(37) a. Michael is close to London.

b. Michael is close to some part of London.

There is little doubt that sentence (37a) entails (37b). However, whether there is also an entailment in the other direction is less evident. Suppose that Michael is in London. In such a case there are surely one or more parts of London that Michael is close to, hence sentence (37b) is true and acceptable, even if somewhat uninformative. By contrast, when we know that Michael is in London it would be very odd to assert sentence (37a). We attribute this oddity of (37a) not to mere falsity of the assertion, but to a presupposition failure. In general terms, we assume that any sentence of the form $A$ is close to $B$ presupposes that $\mathrm{A}$ is outside $\mathrm{B}$, but does not assert it. As evidence for this claim consider the following examples, based on the presupposition tests from von Fintel (2004).

(38) a. A: Mary lives close to her parents' home.

B: Hey, wait a minute. I had no idea that she has left their home.

b. I hope that Mary lives close to her parents' place.

c. If Mary is living close to her parents, she has a chance of having her laundry done fairly regularly.

Using von Fintel's tests, we take the examples in (38) as support for our assumption about the outside presupposition of close to. In (38a), B's reaction indicates that A's utterance presupposes that Mary lives out of her parents' home, rather than asserting this claim. Further, in (38b) the utterance does not express a hope that Mary doesn't live with her parents, but rather presupposes it as a fact. Lastly, in (38c) the statement that Mary does not live with her parents gets projected out of the conditional's antecedent,

\footnotetext{
8 Our use of (35b) for deriving the last equivalence in (36) relies on an assumption (cf. (34)) that $m$ is outside the region $\bigcup\{\operatorname{LOC}(x): x \in \mathbf{g s}\}$. This assumption becomes linguistically more pregnant in our treatment of $\operatorname{DIST}_{\mathrm{M} \downarrow}$ locatives, as discussed below.
} 
which indicates that this claim is a presupposition of the antecedent rather than part of the assertion.

Getting back to sentences (37a-b), we have concluded that the two sentences are not conversationally equivalent: sentence (37a) presupposes that Michael is outside London whereas (37b) does not. (37b) only presupposes that Michael is outside some or other part of London. Incidentally, this is true in all situations, even when Michael is in London. The conclusion is that in situations where Michael is outside London, both sentences in (37) can be safely asserted, since all their relevant presuppositions are met. Furthermore, in such cases there is no truth-conditional difference between the two sentences: if Michael is outside London then he is close to London if and only if he is close to some part of the city. This illustrates what von Fintel (1999) calls Strawson-entailments: cases where an entailment holds between sentences provided that all their presuppositions are satisfied. Accordingly, we say that sentences (37a) and (37b) are Strawson-equivalent.

Against this background, let us now consider the denotations of the locatives close to and at most $20 \mathrm{~km}$ from that are derived from definition (31) for the sets of distances $[0, r)$ and $[0,20]$ respectively. For any region $A$ and point $x \notin A$, we have:

$$
\begin{array}{ll}
\operatorname{ClOSE} \_t \mathrm{TO}(x, A) & \Leftrightarrow \operatorname{DiST}_{[0, r)}(x, A) \Leftrightarrow \mathbf{d}(x, A)<r \\
\text { AT_MOST_20KM_FROM }(x, A) & \Leftrightarrow \operatorname{DiST}_{[0,20]}(x, A) \Leftrightarrow \mathbf{d}(x, A) \leq 20
\end{array}
$$

In words: the relation CLOSE_TO holds between the region $A$ and a point $x$ outside $A$ if the distance between $x$ and $A$ is smaller than some specified 'small' distance $r$; AT_MOST_20KM_FROM holds if the distance between $x$ and $A$ is at most $20 \mathrm{~km}$. It should be noted that the intervals $[0, r)$ and $[0,20]$ are both downward-monotone within the set of non-negative reals. Further, we should note that since definitions (39) and (40) are based on definition (31), they are only specified for cases in which the point $x$ is not in $A$, i.e. the outside presupposition of the corresponding sentence holds.

Now we observe, in contrast to (34), that for any set $R$ of real numbers that is downward-monotone as in (39) and (40), the following fact holds similar to fact (24b) about INSIDE locatives.

(41) Let $R$ be an downward-monotone set of distances. For any collection of regions $\mathcal{A}$ and a point $x \notin \cup \mathcal{A}$, we have:

$$
\operatorname{DisT}_{R}(x, \cup \mathcal{A}) \Leftrightarrow \exists A \in \mathcal{A} \cdot \operatorname{Dist}_{R}(x, A)
$$

In words: when $R$ is downward-monotone, the distance between the union of regions in $\mathcal{A}$ and a point $x$ outside this union is in $R$ iff the distance between $x$ and some region $A$ in $\mathcal{A}$ is in $R$ (cf. (22a)). Specifically in our examples of DISTM $\downarrow$ locatives, we get:

$$
\begin{aligned}
& \text { a. } \quad \operatorname{Close} \_ \text {to }(x, \cup \mathcal{A}) \quad \Leftrightarrow \exists A \in \mathcal{A} \text {.CLose_to }(x, A) \\
& \text { b. } \quad \text { AT_MOST_20KM_FROM }(x, \cup \mathcal{A}) \Leftrightarrow \exists A \in \mathcal{A} \text {.AT_MOST_20KM_FrOM }(x, A)
\end{aligned}
$$

These facts explain why sentences like (37a) and (37b) are judged to be equivalent under the presupposition of (37a) that Michael is outside London. 
Moving back to indefinites, we see that given the PEH, facts (42a) and (42b) also explain the pseudo-existential interpretation of locative indefinites with DIST ${ }_{\downarrow}$ locatives. For instance, let us reconsider sentence (29b), restated in (43a) below, and its pseudo-existential interpretation in (43b).

(43) a. Michael is at most $20 \mathrm{~km}$ from a gas station.

b. Michael is at most $20 \mathrm{~km}$ from $\operatorname{somE}_{\exists}$ gas station.

Our property-based treatment of sentence (43a) leads to the following analysis of this sentence.

$$
\begin{array}{ll}
\text { Asserted: } & \text { AT_MOST_20KM_FROM }(m, \operatorname{LOC}(G S)) \\
\text { Presupposed: } & \text { OUTSIDE }(m, \operatorname{LOC}(G S))
\end{array}
$$

Whenever the presupposition in (44) holds, the definition of OUTSIDE in (21) means that the point $m$ is not in the gas station eigenspace LOC $(G S)$, or, in terms of the PEH:

$$
m \notin \cup\{\operatorname{LOC}(x): x \in \mathbf{g s}\}
$$

Accordingly, we get:

$$
\begin{array}{ll}
\text { AT_MOST_20KM_FROM }(m, \operatorname{LOC}(G S)) & \triangleright \text { assertion-part of (43a) } \\
\Leftrightarrow \text { AT_MOST_20KM_FROM }(m, \bigcup\{\operatorname{LOC}(x): x \in \mathbf{g s}\}) & \triangleright \text { by PEH } \\
\Leftrightarrow \exists x \in \text { gS.AT_MOST_20KM_FROM }(m, \operatorname{LOC}(x)) & \triangleright \text { by (42b) }
\end{array}
$$

The last equivalence follows from the general property (41) of DISTM $\downarrow$ locatives, whose condition $x \notin \cup \mathcal{A}$ holds here thanks to the outside presupposition in (45).

So far we have only considered monotone distal locatives and seen how they support pseudo-existential and pseudo-universal interpretations. Let us now move on to cases where the distance predicate within a distal locative is non-monotone. In such cases the interpretations that emerge cannot simply be described as 'existential' or 'universal'. Sentence (47) below illustrates this point.

(47) Michael is (exactly) $20 \mathrm{~km}$ from London.

The interpretation of (47) is non-existential because in a situation where some part of London is $20 \mathrm{~km}$ from Michael, the sentence may still be false if there is another part of London at a shorter distance. The interpretation of (47) is also non-universal, since the sentence may easily be true when many parts of London are at a distance of more than $20 \mathrm{~km}$ from Michael. The actual interpretation of sentence (47) is paraphrased below.

(48) The part of London that is closest to Michael is $20 \mathrm{~km}$ away from him.

On the background of the discussion above, this interpretation of (47) is hardly surprising. We associate the non-monotone distance predicate exactly $20 \mathrm{~km}$ with the singleton set $\{20\}$. The corresponding distal locative is accordingly defined below.

$$
20 \mathrm{KM} \_ \text {FROM }(x, A) \Leftrightarrow \operatorname{DiST}_{\{20\}}(x, A) \Leftrightarrow \mathbf{d}(x, A)=20
$$


According to this analysis, sentence (47) makes the distal assertion $\mathbf{d}(m, L)=20$, which requires that the closest point in $L$ is $20 \mathrm{~km}$ away from $m$, as intuitively required. This requirement is not simply existential: it is not satisfied if there are points in $L$ less than $20 \mathrm{~km}$ from $m$. Nor is it universal: it may be satisfied when there are points in $L$ more than $20 \mathrm{~km}$ from $m$.

A similar behavior is observed with locative indefinites, as in the following sentence.

(50) Michael is (exactly) $20 \mathrm{~km}$ from a gas station.

In our "desert context", where the question under discussion is how likely it is that Michael can continue the race, sentence (50) has an interpretation that is paraphrased as follows.

(51) The gas station that is closest to Michael is $20 \mathrm{~km}$ away from him.

With the considerations above, it is not surprising that this interpretation of sentence (50) cannot be expressed as either existential or universal. To see that statement (51) is not simple existential quantification over gas stations, suppose that one gas station is exactly $20 \mathrm{~km}$ from Michael, but there is also another gas station that is nearer to Michael's location, say at a distance of $10 \mathrm{~km}$. In the given context this situation makes the prominent interpretation of sentence (50) false, although the existential requirement ('Michael is $20 \mathrm{~km}$ from some $\exists$ gas station') is satisfied. Further, interpretation (51) is not universal, since it allows many gas stations to be at larger distances than $20 \mathrm{~km}$.

We conclude that sentence (50) has a prominent non-existential interpretation, which is furthermore non-universal. Our PEH-based analysis correctly derives this interpretation, as formalized below.

(52) 20KM_FROM $(m, \operatorname{LOC}(G S))$

By definition (49) of the distal relation 20KM_FROM, we get the following analysis.

$$
\mathbf{d}(m, \operatorname{LOC}(G S))=20
$$

In words: the distance between Michael's location and the location of the property GS is exactly $20 \mathrm{~km}$. This means that the closest point in the region $\operatorname{LOC}(G S)$ is $20 \mathrm{~km}$ from the point $m$. Thus, there must be some gas station $20 \mathrm{~km}$ from Michael, and every other gas station is at a distance that is greater or equal to $20 \mathrm{~km}$. This is the interpretation attested for sentence (50) in the given context.

We may, if we like, restate the same interpretation of sentence (50) in terms of quantification over entities, as in the following sentence.

(54) Michael is at most $20 \mathrm{~km}$ from $\operatorname{SOME}_{\exists}$ gas station and at least $20 \mathrm{~km}$ from EvERY $_{\forall}$ gas station.

The paraphrase (54) is equivalent to (51). However, this way of paraphrasing the interpretation of sentence (50) may create the false impression that some or other quantification over entities must always be part of the meaning of locative indefinites. The paraphrase (54) relies on the possibility of presenting the non-monotone distal predicate $20 \mathrm{~km}$ as a boolean combination of the monotone distal predicates at least $20 \mathrm{~km}$ and at most 20km. As in the domain of generalized quantifiers (Thijsse 1983), 
there are also non-monotone numeric predicates that cannot be expressed in this way. One such example is the non-monotone distance predicate an even number of meters. As Thijsse points out, such numeric expressions cannot be described as finite boolean combinations of monotone expressions. Accordingly, we should not expect any finite pseudo-quantificational paraphrase for sentences with $\operatorname{DIST}_{\neg}$ locatives like an even number of meters from. Of course this is a rather artificial example, but it highlights a general lesson: there is no guarantee that sentences with locative indefinites have interpretations that can be paraphrased by using quantification over entities. In the next section we see further evidence for this claim.

\subsection{Projective locatives}

Projective locatives describe positions relative to a specific direction from a landmark. Some examples for this type of locatives are given below.

(55) Projective locatives: above, behind, north of, (to the) left of

Each of the projective locatives in (55) pertains to a different direction from the landmark. The determination of this direction in actual utterances is sensitive to various contextual factors like gravitation or intrinsic properties of the landmark (Herskovits 1986). For instance, the prepositional phrase above John's head describes locations relative to an upward direction from John's head. What is considered 'upwards' may be relative to the earth or to John's posture. For instance, when John lies supine, the locative above John's head may refer to two different directions. Contextual information may prime any of these two directions.

The connection between projective locatives and the part-whole structure of landmarks is often quite complex. This defines any attempt to describe their meanings by using pseudo-quantificational paraphrases as we have so far done in our discussion of topological and distal locatives. The following two sentences illustrate that in the context of Fig. 1a.

(56) a. The dot is to the left of the line.

b. The dot is to the right of the line.

(a)

(b)

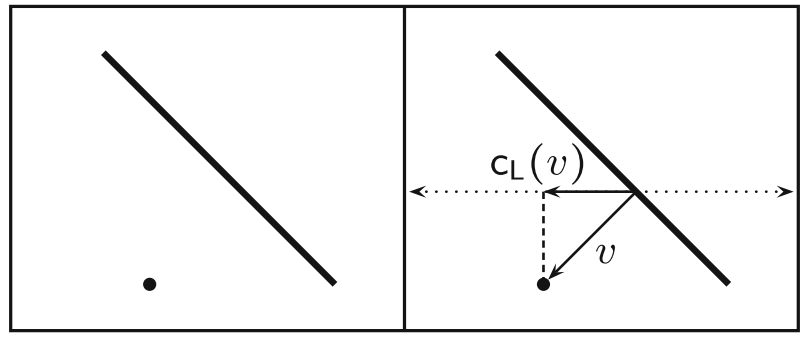

Fig. 1 The dot is to the left of the line 
Sentence (56a) is intuitively true in Fig. 1a, whereas (56b) is false. Following Zwarts and Winter (2000) (henceforth $Z \& W$ ), we capture such effects by appealing to the shortest vector from the landmark to the located object. In Fig. 1b this is the vector $v$ from the line to the dot. As illustrated, $v$ has a non-zero 'left of' component $\mathrm{C}_{\mathrm{L}}(v)$. By contrast, $v$ has zero component in the 'right of' direction. ${ }^{9}$ Accordingly, we adopt the following definition of the projective concept LEFT_OF.

(57) For any region $A$ and point $x \notin A$ :

LEFT_OF $(x, A) \Leftrightarrow$

the shortest vector from $A$ to $x$ has a non-zero 'left of' component

Definition (57), together with the analogous definition for the locative RIGHT_OF, accounts for the contrast between sentences (56a) and (56b) in the context of Fig. 1. It would be hard to describe such contrasts in terms of pseudo-quantification over parts of the line. The reason is that in Fig. 1, the dot is to the left of some parts of the line and to the right of other parts of it. Thus, existential quantification over parts of the line would make (56b) incorrectly true, and universal quantification over parts of the line would make (56a) incorrectly false. The interpretation of sentences like $(56 a-b)$ inherently involves the shape of the objects and their spatial configuration. In general it is hard, if not impossible, to describe meanings of locatives correctly in terms of quantification over parts without taking into account the more 'holistic' aspects of spatial concepts. For this reason, the behavior of projective concepts as in (56) and Fig. 1a is a good test for our analysis of indefinites.

Let us consider the locative indefinites in the following sentences, vis à vis the facts described above.

(58) a. The dot is to the left of a circle.

b. The dot is to the right of a circle.

In the context of Fig. 2a, both (58a) and (58b) may be interpreted as true. This fact is consistent with the existential interpretation of these sentences: in Fig. 2a the dot is to the left of one circle and to the right of another circle. This is accounted for in our proposal by the standard existential analysis, which is assumed as an option for all occurrences of indefinites (Sect. 2). What is more surprising is that in the context of Fig. 2a there is a clear contrast between the two sentences in (58). While sentence (58a) is univocally true in Fig. 2a, sentence (58b) may easily be interpreted as false. Grimm et al. (2014) report experimental results that consistently show this effect with indefinites in projective locatives. In relation to Dutch examples similar to (58), Grimm et al. report that out of 21 Dutch speaking participants, 15 accepted a sentence similar to (58a) as true in a situation like Fig. 2a. By contrast, only 5 of the 21 participants accepted a sentence similar to (58b) as true in this situation. This indicates that in

\footnotetext{
${ }^{9}$ For any non-zero vectors $v$ and $w$ in a vector space $V$, $v$ 's component along $w$ is the (provably unique) vector $c_{w}(v)$ that satisfies $v=c_{w}(v)+u$, where ' + ' is vector addition and $u$ is the (provably unique) projection of the vector $v$ on the set $\perp w$ of vectors orthogonal to $w$ (see e.g. Lang 1977, p. 134). Interpreted in these terms, Fig. 1b demonstrates that $v$ has a non-zero component along any non-zero vector in the 'left' direction.
} 
(a)

(b)

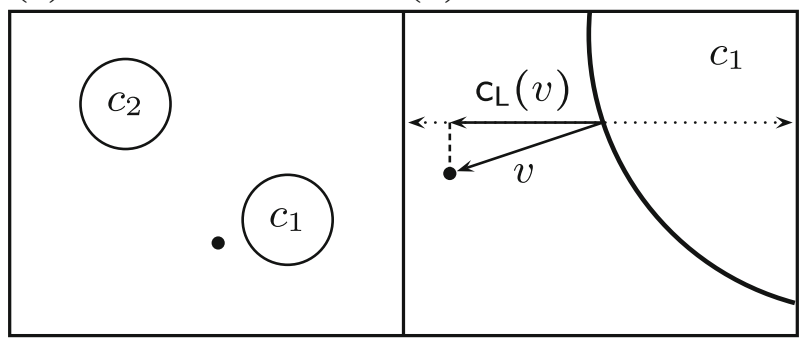

Fig. 2 The dot is to the left of a circle

the context of Fig. 2a, a non-existential strategy is more prominent in (58b) than the existential strategy.

The PEH offers a direct explanation of this non-existential strategy. According to the $\mathrm{PEH}$-based analysis, sentence (58a) has a reading paraphrased as in (59a), whereas (58b) has the reading in (59b).

(59) a. The dot is to the left of the area occupied by the circles.

b. The dot is to the right of the area occupied by the circles.

The PEH-based paraphrase (59a) of (58a) is true in Fig. 2a according to the same principles that make sentence (56a) true in Fig. 1. Analogously, the PEH-based paraphrase (59b) of (58b) is false in Fig. 2a similarly to (56b) in Fig. 1. More formally, consider our PEH-based analysis of (58a) in (60) below. The notation CIRC and circ is for the property denotation of the noun circle and for its set extension, respectively. The notation $d$ is for the eigenspace of the dot.

$$
\begin{aligned}
& \operatorname{LEFT} \_ \text {OF }(d, \operatorname{LOC}(C I R C)) \\
& \triangleright \text { property-based analysis of (58a) } \\
& \Leftrightarrow \text { the shortest vector from } \operatorname{LOC}(\text { CIRC) to } d \text { has a non-zero 'left of' } \\
& \text { component } \\
& \triangleright \text { by definition (57) } \\
& \Leftrightarrow \text { the shortest vector from } \cup\{\operatorname{LOC}(x): x \in \operatorname{circ}\} \text { to } d \text { has a non-zero } \\
& \text { 'left of' component } \\
& \triangleright \text { by PEH }
\end{aligned}
$$

In Fig. 2a we have circ $=\left\{c_{1}, c_{2}\right\}$, and the closest circle to $d$ is $c_{1}$. Therefore, the shortest vector from the eigenspace of the indefinite a circle to $d$ is the vector $v$ from $c_{1}$ to $d$. As illustrated in Fig. 2b, this vector has a non-zero left component $\mathrm{c}_{\mathrm{L}}(v)$. Hence the PEH-based analysis of sentence (58a) is true in Fig. 2a. By contrast, the PEH analyzes sentence (58b) as false, since $v$ has a zero right component. According to the assumed derivational ambiguity of indefinites, both sentences also have a true reading in Fig. 2a. This explains why some speakers accept (58a) and reject (58b) in Fig. 2a, while other speakers accept both sentences. For the first group of speakers, the 
PEH-based strategy is apparently more salient than the existential strategy, hence they accept (58a) but reject (58b) with Fig. 2a. For the second group the existential strategy is more salient, hence they accept both sentences with this figure. For the situation in Fig. 2a, all speakers accepted sentence (58a) as true, as the PEH and the existential strategy converge. We conclude that the $\mathrm{PEH}$, combined with our assumption about the derivational ambiguity of locative indefinites, correctly describes the contrast we found between sentences (58a) and (58b) in the context of Fig. 2a.

\subsection{Modified locatives}

Locative prepositions may appear together with a preceding modificational element within the PP. A modifier of a locative may be a distal predicate as in $10 \mathrm{~km}$ outside or an adverb as in diagonally above. We focus on three types of modified locatives, exemplified in (61) below.

\section{Modified locatives}

a. Topological locative, distal modifier: far outside (of), deep inside (of), two miles within

b. Projective locative, adverbial modifier: diagonally above, straight in front of, right beneath

c. Projective locative, distal modifier: $10 \mathrm{~km}$ north of, deep under, high above, miles west of

We will show further support for the PEH generalization (16) by observing that modified PPs also show close parallelisms between the interpretation of locative indefinites and the interpretation of locative referential NPs. We concentrate on three test cases of PP modification, one for each of the three classes in (61). For each test case we show a simple strategy for interpreting modified PPs with referential NPs. Then we show that the same strategy accounts for non-existential effects with locative indefinites.

\section{Test case 1: distal modification of topological "outside"}

Let us consider the following example.

(62) The hotel is far outside the city center.

In (62) the preposition outside is used topologically, and the PP is modified by the distal predicate far. We can restate such sentences using a conjunction of a topological statement and a distal statement, as in the following paraphrase. ${ }^{10}$

\footnotetext{
10 This conjunctive strategy is not general, and is only made possible by the topological use of outside in (62). However, there is also a projective use for outside: in the stall is diagonally outside the restaurant, the entrance of the restaurant is seen as projecting a direction similar to in front of. As we see below, in such cases PP modification cannot be treated by using conjunction. The conjunctive strategy also fails with modification of topological INSIDE locatives, as in the station is located 50 miles within the park, which means that the station is 50 miles from the border of the park, not from the park itself. For such cases, we also need to use a more general strategy for modifying projective PPs, as discussed below.
} 
(63) The hotel is outside the city center and far from the city center.

Because of the equivalence between (62) and (63), the meaning of the modified PP can be described by using a conjunction between the distal locative and the topological locative. Formally, we define the modified locative concept FAR_OUTSIDE as follows.

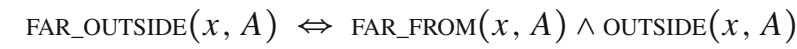

This simple analysis is satisfactory for sentence (62). In such cases the topological concept OUTSIDE adds little semantic content to the purely distal statement the hotel is far from the city center. ${ }^{11}$ Thus, the analysis in (64) together with our account of distal and topological locatives explains why the interpretation of (62) is pseudo-universal, as paraphrased below.

(65) The hotel is far outside EVERY $Y_{\forall}$ part of the city center.

Indefinites within modified OUTSIDE locatives may also give rise to pseudo-universal effects. Let us consider the following examples from the internet.

(66) a. This scene shows Roamer ships encountering a huge, derelict alien city in space, far outside of a star system.

b. The participants were primarily Caucasian and lived at least 25 miles outside of a town of 12,500 or more people.

Sentence (66a), with the upward-monotone distal modifier far, can be paraphrased by "far outside every star system". Similarly, the upward-monotone modifier at least 25 miles in (66b) also gives rise to a pseudo-universal effect: the participants are claimed to live at a distance of at least 25 miles from every big town. The PEH correctly treats such non-existential indefinites by using the conjunctive assumption in (64), and the analysis of indefinites within topological and distal locatives in Sects. 3.2 and 3.3 above. In a similar way, the PEH augmented by the conjunctive analysis account of PP modification analyzes non-existential effects with downward-monotone modifiers and topological outside, as in the following example from the internet.

(67) There's a large selection of campus universities - many built in the 1960susually located a few miles outside of a town or city.

Sentence (68a) below simplifies (67), and can be paraphrased as in (68b).

(68) a. The campus is located a few miles outside of a city.

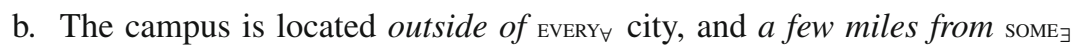
city.

This mixture of a pseudo-universal and a pseudo-existential effect is directly explained by the PEH and the analysis of the modified PP as a conjunction of the OUTSIDE locative and the DIST $\mathrm{M}_{\downarrow}$ locative a few miles from. The OUTSIDE locative contributes the pseudouniversal effect, whereas the DIST $\mathrm{M} \downarrow$ locative makes a pseudo-existential contribution.

11 This distal sentence entails that the hotel is outside the city center. Possibly, this entailment is only a Strawson-entailment, based on an outside presupposition of far from. However, in (62) the assertion of an outside statement is bound to cancel any possible outside presupposition. 
(a)

(b)

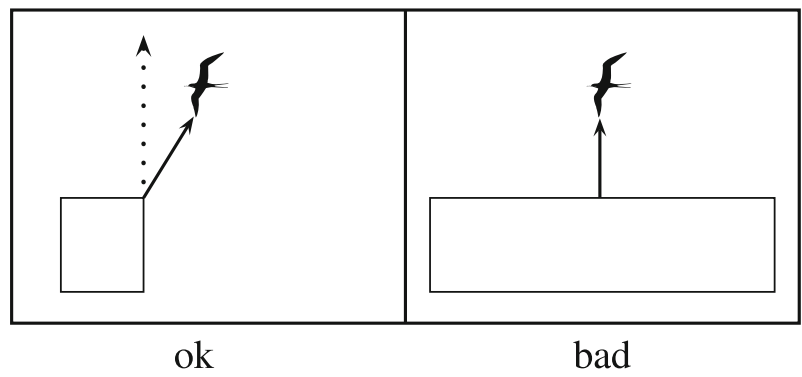

Fig. 3 Diagonally above the house

\section{Test case 2: adverbial modifiers with projective locatives}

The conjunctive analysis is not a general solution to the problem of modified locatives. Matters become more complex when the modified locative is projective. To see that, let us consider sentences (69a-b) below, based on Z\&W's discussion of projective locatives with adverbial modifiers.

(69) a. The dot is straight above the circle.

b. The dot is diagonally above the circle.

The locatives STRAIGHT_ABOVE and DIAGONALLY_ABOVE in (69) cannot simply be defined based on intersection with the denotation of the modifiers straight and diagonally. The reason is that it is hard to conceive of these two adverbials as binary relations on locations. In (69) the modifiers do not simply describe 'straight' or 'diagonal' positions or directions from the circle. What counts as 'straight' or 'diagonal' can only be determined relative to a given direction from the landmark. In (69) the relevant direction ('upward') is determined by the preposition above. As Z\&W note, this kind of phenomenon poses a challenge for any straightforward intersective analysis of the modification in (69). Z\&W account for modified PPs as in (69) using their notion of shortest vectors. As an example for this analysis, we consider the following sentence in the situation of Fig. 3a.

(70) The bird is diagonally above the house.

Sentence (70) is true in Fig. 3a but false (or highly weird) in Fig. 3b. Z\&W's analysis uses the shortest vector between the bird and the house to account for this contrast. In Fig. 3a the shortest vector can truthfully be characterized as 'diagonal with respect to the above direction'. By contrast, in Fig. $3 \mathrm{~b}$ the shortest vector from the house to the bird is not diagonal but straight with respect to the above direction from the house. Summarizing, and skipping some formal details, we restate Z\&W's account of sentence (70) by specifying the locative DIAGONALLY_ABOVE as follows.

$$
\begin{aligned}
& \text { Diagonally_ABOve }(x, A) \\
& \Leftrightarrow \text { the shortest vector from } A \text { to } x \text { is diagonal with respect to the } \\
& \text { above direction }
\end{aligned}
$$


(a)

(b)

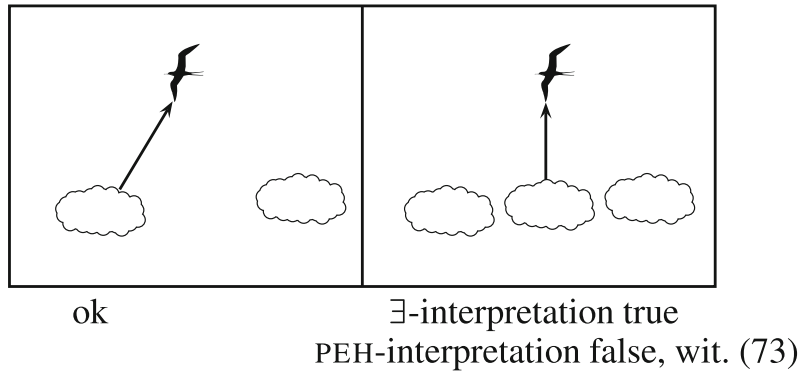

Fig. 4 Diagonally above a cloud

This analysis highlights the fact that the interpretation of sentence (70) cannot be described as pseudo-existential. For the bird to be diagonally above the house, it is not enough for it to be diagonally above some part of the house. If that were the case, sentence (70) should have been equally acceptable in Fig. 3a and b. Rather, for the bird to be diagonally above the house it has to be diagonally above the closest part of the house, as required by Z\&W's account.

Let us now consider the following locative indefinite sentence in the context of Fig. 4a.

(72) The bird is diagonally above a cloud.

In Fig. 4a, sentence (72) is intuitively true. By contrast, in Fig. 4b, the sentence is true under its existential interpretation, but intuitively it also has a non-existential interpretation, under which it is false. To see this in a more natural context, consider the following example, which describes two cases of aerial inflammations. ${ }^{12}$

(73) The first case arises when the inflammation is straight above a thick cloud, the second when it is obliquely above a thick cloud.

Sentence (73) refers to two distinct cases of inflammation. Thus, in the second case, where the inflammation is obliquely above some thick cloud, we expect the first case not to hold, i.e. we expect the inflammation not to be located straight above any other thick cloud. This shows a non-existential effect in the interpretation of the italicized PP in (73): the locative indefinite refers to the position of the inflammation relative to the closest cloud below it. This is the same 'non-existential' behavior we observed in sentence (70), where the part of the house that is relevant for (70)'s truth in Fig. 3a is the part that is closest to the bird.

When we adopt Z\&W's shortest vector account we can readily use the PEH to account for non-existential effects with indefinites as in (72) and (73). Specifically, for (72) we get the following analysis.

12 This example simplifies a more complex description from the book Aristotle's Meteorology and Its Reception in the Arab World (P. Lettinck, Brill, 1999, p. 72): "The colours in the sky arise because light from the inflamed 'fire' is seen either refracted or reflected against the thicker air. The first case arises when the inflammation is above a cloud that is not too thick, the second when it is obliquely above a thick cloud". 
(a)

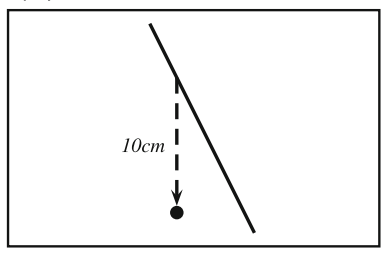

$10 \mathrm{~cm}$ below the line (b)

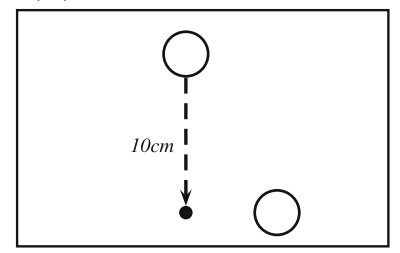

$10 \mathrm{~cm}$ below a circle (c)

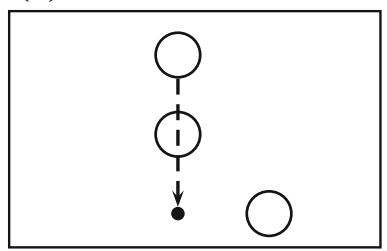

$\# 10 \mathrm{~cm}$ below a circle

Fig. 5 Modified locatives with a referential NP and with an indefinite

$$
\begin{aligned}
& \text { DIAGONALLY_ABOVE }(b, \operatorname{LOC}(\text { CLOUD })) \\
& \Leftrightarrow \text { shortest vector from LOC }(\text { CLOUD }) \text { to } b \text { is diagonal w.r.t. 'above' direction } \\
& \quad \triangleright \text { by Z\&W's analysis (71) }
\end{aligned}
$$
'above' direction

$$
\triangleright \text { by } \mathrm{PEH}
$$

$\Leftrightarrow$ the shortest vector to $b$ from the cloud in cloud that is closest to $b$ is diagonal w.r.t. the 'above' direction

This analysis of sentence (72) is true in Fig. 4a but false in Fig. 4b. Accordingly, it is useful for describing the behavior of such sentences in contexts like (73).

\section{Test case 3: distal modifiers with projective locatives}

As illustrated in (61c) above, projective locatives are often modified by distal predicates. Let us now consider the following sentence, with a locative referential NP, in the situation of Fig. 5a.

(75) The dot is $10 \mathrm{~cm}$ below the line.

Sentence (75) is intuitively judged as true in Fig. 5a. As noted in Sect. 3.4, similar situations support 'non-existential' interpretations of such sentences with projective locatives. Also sentence (75) cannot be paraphrased by stating that the dot is $10 \mathrm{~cm}$ below some part of the line. In Sect. 3.4 we saw how the PEH makes a connection between this fact and non-existential effects with indefinites in simple projective locatives. Now let us observe a similar point with respect to indefinites in modified projective locatives. This is illustrated by the following sentence.

(76) The dot is $10 \mathrm{~cm}$ below a circle.

In Fig. 5b, sentence (76) is intuitively true. This is not simply an existential effect: sentence (76) is much less felicitous as describing Fig. 5c, where another circle intervenes between the dot and the circle that is $10 \mathrm{~cm}$ above it. Thus, this is another illustration for the non-existential interpretation of indefinites within projective PPs, discussed in Sect. 3.4.

However, unlike the projective constructions treated in Sect. 3.4, we cannot use Z\&W's shortest vector analysis for either sentence (76) or (75). Reconsider sentence 
(75). While this sentence is judged as true in Fig. 5a, Z\&W's analysis incorrectly treats it as false. This is because the shortest vector from the line to the dot in Fig. 5a does not point downwards, and it is shorter than $10 \mathrm{~cm}$. We can solve this problem for Z\&W's account by using the following analysis of sentence (75).

(77) The shortest vector to the dot that points downward from the line is $10 \mathrm{~cm}$ long.

This statement is true in Fig. 5a, as intuitively required for sentence (75). When analyzing sentence (76) we use the same method, now employing the PEH analysis of the indefinite. We get the following analysis.

(78) The shortest vector to the dot that points downward from eigenspace of the circles is $10 \mathrm{~cm}$ long.

This statement is true in Fig. 5 b but false in Fig. 5c, as intuitively required for sentence (76).

Other cases of projective PPs modified by distal predicates show non-existential effects similar to sentence (76). The following examples from the internet illustrate that.

(79) a. The VFR rules say that you must stay at least 500 feet below a cloud, more than 1000 feet above a cloud, and more than 2000 feet to the side of a cloud. (online network simulating real world flying and air traffic control)

b. The pilot reported that he had been flying approximately 500 to 800 feet below a cloud layer that he estimated to be broken at 4,000 agl. (website on aviation accidents, $a g l=$ above ground level)

Similarly to sentence (76), also the sentences in (79a-b) have prominent non-existential interpretations: the indefinites do not refer to distances to an arbitrary cloud, but to distances to a cloud (or a cloud layer) that is closest to the located object. Therefore, (79a-b) are not accounted for by a simple existential analysis. Rather, they are treated by the same revision of Z\&W's analysis that we used for sentence (76). More details on this necessary revision are deferred to future work.

\subsection{Summary: the PEH and locative indefinites}

In this section we have seen various non-existential effects with locative indefinites. Some of these effects are pseudo-universal, with the topological locative OUTSIDE and upward-monotone distal locatives like FAR_FROM. However, in many other cases the non-existential effects with locative indefinites are more complex. This is especially the case with non-monotone distal locatives, projective locatives and modified locatives. As we have seen, whenever locative indefinites exhibit non-existential effects we find locative sentences with referential NPs that show similar effects with respect to the part-whole structure of entities. These correlations are expected by the PEH generalization in (16). They help us to explain away the apparently recalcitrant behavior of locative indefinites as a pseudo-quantificational effect, which follows directly from standard geometrical assumptions about locatives and the treatment of indefinites as denoting properties under the Property-Eigenspace Hypothesis. The PEH does 
not stand in opposition to the traditional existential/specific account, which we still assume as one of the possible derivations with property-denoting indefinites. The PEH does stand in opposition to any approach that strives to account for all interpretations of locative indefinites as following from existential quantification over entities. In the next section we discuss one possible attempt to follow such a line, and show reasons to reject it.

\section{What makes pure existential accounts undesirable?}

An alternative to our property-based approach might be to assume that locative indefinites only denote existential quantifiers, but lead, by some means or other, to non-existential effects at the sentence level. In this section we briefly consider a possible version of this idea, suggested to us by an anonymous $L \& P$ reviewer. The reviewer proposes to analyze certain locatives as containing a covert negation operator that takes scope over an existential quantifier. After elaborating on this idea, we argue that it is conceptually inelegant and show that it is insufficient as a general theory of the phenomena described in Sect. 3.

\subsection{Introducing covert negation in locatives}

The idea to add covert negation to the analysis of locatives is inspired by classic logical equivalences as in (80) below, where a negated existential behaves like a universal quantifier over a negated proposition.

(80) $\neg \exists x . P(x) \Leftrightarrow \forall x . \neg P(x)$

The equivalence in (80) is standardly used for explaining scopal interactions of indefinites with negation, as in sentence (81) below.

(81) John did not see a lion.

Sentence (81) has an existential/specific interpretation ('there's a lion that John didn't see') and a universal interpretation ('for each lion $x$, John didn't see $x$ '). These two interpretations are traditionally analyzed as two different readings of sentence (81) under structural ambiguity. This is illustrated by the following simplified syntacticsemantic analysis of the ambiguity.

$$
\begin{array}{ccc}
\text { a. John [[did not see] } \operatorname{some}_{\exists} \text { lion] } & \exists x \in \operatorname{lion} . \neg \operatorname{see}(\mathbf{j}, x) \\
\text { b. John [did not [see } \operatorname{some}_{\exists} \text { lion]] } & \neg \exists x \in \operatorname{lion} . \operatorname{see}(\mathbf{j}, x) \\
& \Leftrightarrow \forall x \in \operatorname{lion} . \neg \operatorname{see}(\mathbf{j}, x)
\end{array}
$$

In analysis (82a) the existential quantifier takes scope over the negated verb. By contrast, in (82b), the existential quantifier takes scope below negation, which leads to a universal effect at the sentential level, as intuitively required.

An anonymous $L \& P$ reviewer suggests to use a similar line for treating locative indefinites as in our initial example (1a), which is reproduced below. 
(83) Michael is far from a gas station.

Sentence (83) does not contain overt negation. However, suppose that the locative far from in (83) is considered synonymous with the expression not close to, and is decomposed at some level of the syntactic-semantic analysis into this negated form. With the necessary lexical and syntactic assumptions, sentence (83) might then be treated as scopally ambiguous similar to sentence (81). More concretely, using decomposition we may treat sentence (83) as having the following two analyses.
a. Michael is [[not close to] $\operatorname{some}_{\exists}$ gas station]
$\exists x \in$ gs. $\neg$ close_to $(\mathbf{m}, x)$
b. Michael is [not [close to $\operatorname{some}_{\exists}$ gas station]]
$\neg \exists x \in$ gs.close_to $(\mathbf{m}, x)$ $\Leftrightarrow \forall x \in$ gs. $\neg$ close_to $(\mathbf{m}, x)$

In this analysis, the indefinite a gas station is treated as existential under both readings of the sentence, but one of the readings is universal due to the scope of the covert negation operator over the existential quantifier.

\subsection{Arguments against the covert negation analysis of locatives}

There are various reasons for rejecting the covert negation idea as a general account of locative indefinites. These reasons concern problems with non-universal interpretations, the massive decomposition required, unclear relations with other decompositional approaches, and the distribution of a/some contrasts.

Non-universal locative indefinites. Negating an existential quantifier results in universal quantification. However, some of the effects reviewed in Sect. 3 are neither existential nor universal. Covert negation would not help in treating these examples. ${ }^{13}$ Thus, a proponent of the idea that indefinites unambiguously denote existential quantifiers might try to capture contrasts as in (58a-b) by augmenting the system with some additional principles, e.g. a proximity principle that would give primacy to the circle in Fig. 2 that is closer to the dot. Such a principle would stipulate one prediction of the PEH-based analysis and incorporate it into the semantic analysis of existential indefinites, without giving any support to the decompositional approach itself. Similar remarks hold with respect to the non-universal interpretations of non-monotone modified locatives presented in Sect. 3.5.

The contrast between ' $a$ ' and 'some'. As we saw, some indefinites in PPs like far from some gas station only show existential/specific interpretations. The decompositional approach may block the universal analysis by using the common assumption that some is a positive-polarity item, i.e. an expression that cannot appear in the scope of negation (Szabolcsi 2004; Giannakidou 2011). This standard assumption would not help to analyze some indefinites in constructions like the dot is to the left of/10cm below

\footnotetext{
13 For the contrast (58a-b) in the context of Fig. 2, let us suppose that we tried to analyze to the left of a circle as not to the right of $\mathrm{SOME}_{\exists}$ circle. This would only derive an incorrect universal analysis for (58a). Furthermore, such an analysis would not account for the same contrast in the symmetric situations with right of.
} 
some circle, where again, only the existential interpretation is available. Since such cases cannot be analyzed by covert negation, the decompositional approach would need a separate principle for explaining the difference between some and $a$ when appearing with projective locatives like left of and right of.

Decomposition beyond 'far from'. As we saw in Sect. 3.3, upward-monotone distal locatives like at least $20 \mathrm{~km}$ from show the same (pseudo-)universal interpretations as far from. In the decompositional approach, this would mean that also these locatives should be analyzed as containing covert negation. For instance, the locative at least $20 \mathrm{~km}$ from a church would have to be decomposed into not less than $20 \mathrm{~km}$ from a church. Further, to capture the mixed existential/universal effects we saw with nonmonotone distal locatives, we would need to decompose the locative exactly $20 \mathrm{~km}$ from a church into at most $20 \mathrm{~km}$ from a church and not less than $20 \mathrm{~km}$ from a church. A theory that would try to adopt such decompositional stipulations for modified numerals would be unnecessarily complicated and inelegant.

Modification of decomposed locatives. Yet another complication for the decompositional approach is the treatment of modified locatives like $3 m$ outside. To capture the pseudo-universal behavior of outside, as described in Sect. 3.2, the decompositional approach would have to decompose it into not inside. However, the form $3 \mathrm{~m}$ not inside is ungrammatical. To analyze such strings, the decompositional analysis would have to stipulate ad hoc semantic mechanisms only in order to analyze decomposed representations that the theory generates.

Selecting the decomposed item in antonymous pairs. Even in relation to the apparently successful decomposition of sentence (83) above there are some unresolved theoretical questions. While some works have assumed decomposition of adjectives, we are not aware of any independent motivation for the particular decomposition that the anonymous reviewer suggests for sentence (83). For instance, Büring (2007) proposes to decompose negative adjectives like short into little long. Heim (2008) argues against this proposal. However, even ignoring Heim's critique, we do not see how to reconcile the reviewer's suggestion with Büring's proposal. In the decompositional treatment that was suggested to us, the locative containing the positive adjective far is decomposed by using the adjective close. By contrast, in Büring's analysis the adjective close would be decomposed as little far, whereas the morpheme far would be left intact. Thus, as far as we can see, the decompositional analysis that the reviewer suggested for sentence (83) is inconsistent with Büring's proposal.

Summary. We have seen that the decompositional approach to locative indefinites must be limited to those cases that are interpreted universally. These are only a small subset of the non-existential effects that we have studied with locative indefinites. More complicated assumptions must be introduced to account for other non-existential effects. Furthermore, even with the upward-monotone distal locatives, which are interpreted universally, the decompositional approach would have to be quite cumbersome, and in conflict with other decompositional approaches to adjectives.

A note on NPIs. A conceivable argument in favor of the covert-negation approach is the distribution of negative polarity items, as illustrated by contrasts like far from 
(\#close to) any car (Iatridou 2003, 2007). Such contrasts might be accounted for by the reviewer's proposal that far from, but not close to, involves a covert negative element. However, this would not constitute an advantage over the PEH. As we show in Sect. 5 , our proposal directly accounts for such contrasts with NPIs by relying on the same locative denotations we used above for far from and close to, which are distinguished in terms of their monotonicity properties. These properties are standardly used for explaining the distribution of NPIs without any postulation of covert negation.

\section{Further issues}

In this section we briefly remark on some broader implications of the semantics we have proposed for locative indefinites. We start out with two possible extensions of the framework for treating related problems with collective plural descriptions and with negative polarity items. Then we briefly remark on some open problems regarding property indefinites and part-whole structures, which are also of much relevance to the analysis of locative indefinites.

\subsection{Treating locative plurals and locative NPIs}

\subsubsection{Locative collections and eigenspace convexity}

Our treatment of pseudo-quantificational effects with locative indefinites has gained support from the similar behavior of entity-referring NPs. Our initial examples were the equivalences between far from London and 'far from every part of London', and between close to London and 'close to some part of London'. Part-whole structure is also manifested in the compositional semantics of NPs involving collective reference. Let us consider for instance the noun phrases the mountains and the mountain range in the following examples.

(85) a. The house is far from the mountains.

(86) a. The house is close to the mountains. b. The house is far from the mountain range.

b. The house is close to the mountain range.

The sentences in (85) mean that the house is far from every mountain (in the mountain range). By contrast, in (86a-b), only one of the mountains has to be close to the house. Thus, like singular indefinites, also referential plurals (the mountains) and referential group terms (the mountain range) show the existential/universal variability we observe with 'simple' singular NPs like London, the city or Sierra Nevada. In all these cases, geometric features of the locative uniformly determine the pseudo-quantificational interpretation. We conclude that referential NPs, both singular and plural, are assigned eigenspaces that partake in a uniform way in the spatial interpretation of locative sentences. From the point of view of theories of plurality this uniformity is hardly surprising: both referential plurals and singular group terms are often treated as entitydenoting, similar to referential singular NPs (Winter and Scha 2015). 


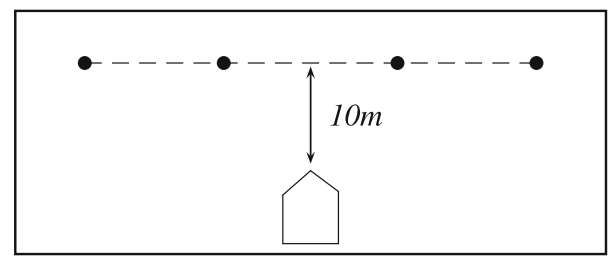

Fig. 6 The house is $10 \mathrm{~m}$ from the (row of) utility poles/\#10m from a utility pole

We might assume that the assignment of eigenspaces to singular and plural entitydenoting NPs is identical to how eigenspaces are assigned to property-denoting indefinites. However, in one respect locative indefinites are different from locative referential NPs. According to the PEH, when a property $P$ is assigned an eigenspace, this has to be the union of the eigenspaces of entities in $P$ 's extension. However, using a completely analogous analysis for locative referential NPs would not be fully accurate. Locative referential NPs have a tendency to show an 'atomic' behavior, which sets them apart from property-denoting indefinites. To see what we mean by that, let us consider the following examples in the context of Fig. 6.

(87) a. The house is (exactly) 10m away from the (row of) utility poles.

b. The house is (exactly) $10 \mathrm{~m}$ away from a utility pole.

In Fig. 6, the pole that is closest to the house is more than $10 \mathrm{~m}$ away from it. Despite this fact, the sentences in (87a) may be interpreted as true: we may think of the eigenspace of the description the (row of) utility poles as including the electric cable that the poles support, or even just an imaginary line between them. This geometric 'extension' procedure is not at work in sentence (87b), which must be interpreted as false in Fig. 6. Like Zwarts and Winter (2000), we propose that in sentence (87a), the eigenspace of the landmark is the convex hull of the poles' locations, here the line segment between them. ${ }^{14}$ This means that the eigenspace of the plural or group term is a proper superset of the union of eigenspaces for the single poles. This 'geometric extension' is a well-known effect with spatial expressions: in a similar way, sentences like the dot is inside the ring may be interpreted as true when the actual material of the ring does not contain the dot, but the dot is surrounded by the ring. ${ }^{15}$ As we saw, a parallel 'geometric extension' procedure is not licensed with locative indefinites as in (87b). This contrast shows a clear difference between how eigenspaces are assigned to entities and to properties. We cannot further study this difference here, but we believe

\footnotetext{
14 The convex hull of a region $A$ (in a Euclidian space) is the smallest region $B$ containing $A$, such that for every two points $x, y \in B$, the line segment between $x$ and $y$ is contained in $B$. In the example above, the convex hull of the finite set of poles on a line is the line segment connecting them. In the example the dot is inside the ring below, the convex hull of an annular geometric shape is the outermost circle in that annulus. 15 In 'the fox is inside the ring of hounds', the fox is surrounded by the hounds. By contrast in 'the bug is inside the bagel', the bug is most likely to be within the bagel's eigenspace. What is considered insideloutside non-convex shapes like rings is partly decided by contextual factors. For further examples see Herskovits (1986).
} 
that finding more contrasts of this sort would help to understand better the use of eigenspaces in spatial semantics. ${ }^{16}$

\subsubsection{Anti-additivity and negative polarity items}

We have seen two classes of locatives that give rise to pseudo-universal interpretations: upward-monotone distal locatives like far from and more than $10 \mathrm{~km}$ from, and topological locatives like outside (of). These two classes of locatives show clear contrasts with downward-monotone distal locatives (close to, less than $10 \mathrm{~km}$ from) and the topological locative inside, which give rise to pseudo-existential interpretations. The following equivalences reiterate these contrasts.

(88) a. Michael is far from London $\Leftrightarrow$ Michael is far from every part of London.

b. Michael is close to London $\Leftrightarrow$ Michael is close to some part of London.

(89) a. Michael is outside London $\Leftrightarrow$ Michael is outside every part of London.

b. Michael is inside London $\Leftrightarrow$ Michael is inside some part of London.

In formal terms, we have accounted for such contrasts using the following equivalences, where $x$ is a point, $A$ is a region, and $\mathcal{A}$ is a set of regions (cf. (24), (35a), (42a)).

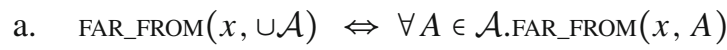

$$
\begin{aligned}
& \text { b. } \quad \operatorname{ClOSE} \_ \text {to }(x, \cup \mathcal{A}) \Leftrightarrow \exists A \in \mathcal{A} \text {. } \operatorname{Close}_{-} \text {to }(x, A) \\
& \text { a. } \operatorname{Outside}(x, \cup \mathcal{A}) \Leftrightarrow \forall A \in \mathcal{A} \operatorname{doutside}(x, A) \\
& \text { b. } \operatorname{Inside}(x, \cup \mathcal{A}) \Leftrightarrow \exists A \in \mathcal{A} \cdot \operatorname{Inside}(x, A)
\end{aligned}
$$

In the linguistic literature, functions that support equivalences with universal statements as in (90a) and (91a) are known as anti-additive functions. Functions that support equivalences with existential statements like (90b) and (91b) are often called additive. In more precise terms, the equivalences in (90a)-(91b) illustrate (anti-)additivity on the second argument of the locative relation. A simple fact is that anti-additive functions are downward monotone, whereas additive functions are upward monotone. ${ }^{17}$

Anti-additivity, and more generally downward-monotonicity, are often used when accounting for the distribution of negative polarity items (NPIs, see Fauconnier 1975;

\footnotetext{
16 For another relevant contrast between the two uses of eigenspaces, consider the sentence the circle is inside the rectangles, which can be true when the circle is contained in the union of the rectangles, but not in any single rectangle. By contrast, the circle is inside a rectangle is purely existential and cannot be true in such a situation. Our proposal here does not account for such contrasts, since the located object the circle is treated as a point, which cannot be contained in a finite union of rectangles unless it is contained in one of them.

17 Standardly, we say that a function $f: \wp(E) \rightarrow\{0,1\}$ is downward monotone if for all $A, B \subseteq E$ : $A \subseteq B \Rightarrow f(B) \leq f(A)$, and upward monotone if for all $A, B \subseteq E: A \subseteq B \Rightarrow f(A) \leq f(B)$. The ' $\Rightarrow$ ' direction in (90a) and (91a) shows that FAR_FROM and OUTSIDE are downward monotone on their second argument; the ' $\Leftarrow$ ' direction in (90b) (a Strawson-entailment) and (91b) shows that CLOSE_TO and INSIDE are upward monotone on their second argument. Since distal locatives like less than $5 \mathrm{~km}$ from are additive on their second argument, they are upward monotone. However, the distal predicate within the locative, i.e. less than $5 \mathrm{~km}$, is downward monotone. Therefore, somewhat counter-intuitively, we called such distal locatives 'DIST ${ }^{\downarrow} \downarrow$ ' despite their upward-monotonicity.
} 
Ladusaw 1979; Nam 1994; Zwarts 1998; Giannakidou 2011). For NPIs like any and ever, a commonly proposed grammaticality filter is that they should appear in the scope of an anti-additive function, or at least a downward-monotone function. With respect to distal locatives like far from and close to, Iatridou (2003, 2007) discusses contrasts like the following.

(92) Michael is far from (\#close to) any gas station.

With outside and any, many examples like the following are easily found on the web. ${ }^{18}$

(93) Bobby Finger is outside any political niche or anything anyone could call a mainstream media presence.

The site is outside any identified good risk area on the Environment.

I planted a $15 \times 11$ area to use as an orchard, it is outside of any forestry area.

Svalbard is outside of any social security exchange contract.

By contrast, when searching strings like inside any, we most frequently found NPIs licensed by a non-locative downward-monotone function. For instance, in the clause if the test point is inside any of the hole boundaries, the NPI licenser is most likely the conditional. In many other cases of inside any, the item any is used as a freechoice item rather than an NPI, e.g. Melophobia is inside any Humbug pilgrim (for the NPI/FC distinction, see Giannakidou 2001).

Based on our treatment of locative indefinites, anti-additivity/downward-monotonicity of locatives accounts for the pattern observed with NPI licensing: far from/outside (of) $a n y_{\mathrm{NPI}} \mathrm{Vs}$. \#close to/inside any $\mathrm{NPI}_{\mathrm{NI}}$. More evidence for this treatment, here again with indefinites in the scope of the locative, come from the following entailments.

(94) a. Michael is far from a gas station $\Rightarrow$ Michael is far from a big gas station.

b. Michael is close to a gas station $\not$ Michael is close to a big gas station.

(95) a. The school is outside of a metropolitan county

$\Rightarrow$ The school is outside of a big metropolitan county. (cf. (20c))

b. The school is within a metropolitan county

$\not$ The school is within a big metropolitan county.

We analyze the entailments of (94a) and (95a) as involving containment between the extensions of the properties big gas station/gas station and big metropolitan county/metropolitan county, hence also between their respective eigenspaces. With these containment relations, the entailments in (94a) and (95a) are expected because of the downward-monotonicity of far from and outside. By contrast, (94b) and (95b)

\footnotetext{
18 Lisa Matthewson suggests that in some cases there is a difference between outside any sentences like the dog is outside any doghouse and the dog is outside (of) a doghouse, where the latter involves a stronger domain restriction (e.g. the former is more easily acceptable in a context where there are no doghouses around). We believe that this question can only be treated within a theory of NPIs, where domain widening/restriction effects are taken into account. This goes beyond the scope of this paper. Another empirical question that Matthewson raises is whether there is a difference between outside (of) $a$ and far from a in terms of their domain restrictions. We, like Matthewson, believe that the former are more restrictive, but we were not able to come up with decisive tests that would allow us to account for it.
} 
involve upward-monotone locatives, and the entailments are properly blocked by our account. We note that a traditional, wide-scope existential analysis of the indefinites in (94)-(95) would not account for these entailment patterns. For instance, in (94), if there is a gas station $x$ s.t. Michael is far from $x$, it does not follow that there is such a big gas station.

We conclude that both the distribution of NPIs and the entailment patterns with $a$ indefinites support our characterization of anti-additivity with locatives, and more importantly for the purposes of this paper, the analysis of $a$ indefinites as having narrow scope with respect to the locative expression.

\subsection{Loose ends}

\subsubsection{Property-denoting indefinites, the a/some distinction, and indefinite subjects}

As we have seen, locative indefinites with the article $a$ get non-existential interpretations, which the PEH analyzes by using their property denotation. Property-based accounts have also proven useful for analyzing other phenomena with indefinites. These phenomena include generic interpretations, predication constructions, there sentences, intensional transitive verbs, and incorporation phenomena. ${ }^{19}$ Ideally, we should like to know if the current property-based semantics can be part of a unified theory in this large linguistic domain. While this general question goes beyond the scope of this paper, we would like to point out one of its important aspects: the distinction between $a$ indefinites and some indefinites. As we illustrated, some indefinites only show existential/specific interpretations in the locative constructions we studied. In this respect locative indefinites are similar to singular generic indefinites and predicative indefinites. This can be illustrated by the following pairs of sentences.

(96) A/some madrigal is polyphonic. (Lawler 1973)

(97) a. John is a/some man (I know).

b. I consider John a man/\#some man.

(98) Mary is looking for a/some maid.

(99) There is a/some dog in the kitchen.

In (96) the generic interpretation obtains with the $a$ indefinite but not with the some indefinite. In (97) the $a$ indefinite licenses the predication, whereas the some indefinite is ruled out or only licensed under a wide-scope/specific existential interpretation (Doron 1983; Winter 2001). In (98), the $a$ indefinite can be interpreted as either de dicto or de re, whereas the some prefers, or even requires, the de re interpretation. By contrast, in (99), both the $a$ and the some indefinites are licensed and lead to a similar interpretation. This means that the line suggested here is consistent with

\footnotetext{
19 For analyses of these phenomena see: Cohen (2001), Greenberg (2007) on generic interpretations of singular $a$ indefinites; Partee (1987), Winter (2001), Landman (2014) on predicative a/some indefinites; McNally (1998, 2011) on there sentences; Zimmermann (1993), Van Geenhoven and McNally (2005) on intensional transitive verbs; Van Geenhoven (1998), Chung and Ladusaw (2003), Farkas and de Swart (2003) on incorporation.
} 
theories of the phenomena in (96)-(98), which let $a$ indefinites denote properties, but avoid this denotation for some indefinites. However, theories like McNally's (1998), which rely on property denotations of indefinites to also account for their behavior in there sentences, need to have a special account for the lack of the a/some contrast in cases like (99), vis à vis the contrasts in (96)-(98).

A related issue concerns property interpretations of locative indefinites in subject position. To see it, let us consider the contrast between sentences (100) and (101) below in the given contexts. ${ }^{20}$

(100) Context: Michael is driving in the Mojave Desert, moving away from the Las Vegas metropolitan area.

(However,) a gas station where Michael could rest and fuel up is still nearby.

(101) Context: Michael is driving in the Mojave Desert, moving towards the Las Vegas metropolitan area.

(However,) a gas station where Michael could rest and fuel up is still faraway.

In the given contexts there is a clear contrast between (100) and (101). Sentence (100) only has an existential/specific interpretation: one gas station near Michael is enough to make it true. By contrast, sentence (101) requires Michael to be far from all relevant gas stations. Thus, sentences (100) and (101), with a locative $a$ indefinite in the subject position, show the same pseudo-quantificational contrast that we have observed all along in sentences where the locative indefinite is inside the PP. We note that there is no a priori reason to think that it is the context in (100) and (101) that favors the pseudo-existential or pseudo-universal interpretation. As far as the contexts go, the situation might have been the other way around. The fact that the interpretations are as they are in (100) and (101) is further evidence for the PEH.

Notably, PEH-like effects with indefinite subjects as in (100)-(101) were considerably harder to find than the other pseudo-quantificational effects we studied using indefinites within locative PPs. For simple examples with indefinite subjects, e.g. $a$ gas station is nearby/faraway, speaker judgements are less secure and it is harder to observe contrasts similar to the one between (100) and (101). The modal ('could') and the temporal adverb ('still') make the judgements clearer in (100)-(101). This is unlike the situation with indefinite PPs, e.g. close tolfar from a gas station, where no priming coming from modal or temporal expressions was required. We have no precise semantic proposal that would predict this difference between locative indefinites in subject position and locative indefinites within PPs. Hopefully, on-going work on asymmetries between indefinite subjects and indefinite objects may help to shed more light on this problem (de Hoop and Kramer 2006; Bianchi and Belletti 2014).

\subsubsection{Subpart monotonicity and part-whole structure}

Part-whole relations and spatial relations have attracted much attention in the philosophical literature and in semantic theory (Cruse 1979; Herskovits 1986; Winston et al. 1987; Iris et al. 1988; Moltmann 1997; Casati and Varzi 1999; Johansson 2004).

$\overline{20}$ We thank Heidi Klockmann and Yael Seggev for their help with these examples. 
Without getting into many of the relevant philosophical problems, this paper has concentrated on some linguistic puzzles about locatives and part-whole relations that seem to us of importance for semantic theories of indefinites and plurals. We started our study with subpart relations between descriptions of geographical units. As we saw, being in a locative relation to a complex entity like London has clear implications for locative relations to its subparts. For instance, being far from London entails being far from Islington, by virtue of the subpart relation between these two geographical entities. Further, we have also touched upon subpart relations between entities and collections. One example for this kind of subpart relations is the one between the entity for $M t$. Whitney and the collection of entities denoted by descriptions like the mountains, the mountain range or Sierra Nevada. This kind of subpart relations also affects pseudoquantificational interpretations with locatives. We proposed-and this is the essence of the PEH - that locative relations work similarly with indefinites like $a$ city, which are treated as denoting a property that has its own eigenspace. In this treatment, the entity for a specific city like London stands in a part-of relation to the denotation of the indefinite $a$ city, in much the same way as Islington stands in a part-of relation to London. These different types of sub-part relations support spatial entailments like the following (Iris et al. 1988, p. 435).

(102) Our friend is in Islington $\Rightarrow$ Our friend is in London.

(103) The camp is on Mt. Whitney $\Rightarrow$ The camp is in Sierra Nevada.

The camp is on Mt. Whitney $\Rightarrow$ The camp is in the mountains (of Sierra Nevada).

The camp is on Mt. Whitney $\Rightarrow$ The camp is in the mountain range (of Sierra Nevada).

(104) Max is in London $\Rightarrow$ Max is in a city.

The classification of the relation between London and a city as a 'subpart' relation is the most debatable. This is because entailments as in (104) are also expected by the traditional existential analysis of indefinites. However, while accepting the standard existential analysis as a viable option, we have seen that treating indefinites as property-denoting has linguistic advantages over their unitary treatment as quantifiers. This means that our PEH-based analysis of sentences like Max is in a city in (104) is on a par with more classical accounts of sub-part entailments as in (102) and (103).

Considering all the relations mentioned above as instances of one general subpart relation, our accounts of all of these relations have consistently adopted the following assumption, which Casati and Varzi (1999, p. 15) dub "obvious".

(105) If $y$ is subpart of $x$, then $\operatorname{LOC}(y) \subseteq \operatorname{LOC}(x)$.

In words: when $y$ is a subpart of $x$, x's location must contain $y$. In our terms, this assumption reflects a monotonicity of the eigenspace function LOC with respect to the subpart order on objects (cf. Casati and Varzi 1999, p. 54). From this assumption we conclude: 
(106) If the elements in a set $Y$ are all subparts of $x$, then $\bigcup_{y \in Y} \operatorname{LOC}(y) \subseteq \operatorname{LOC}(x)$.

In our analysis of indefinites, we strengthened this subset relation into an equality whenever $x$ is a property and $Y$ is its extension. Thus, in the PEH we proposed that the location of a property equals the union of its extension's member locations. We leave it for further research to examine if a similar strengthening is also useful for subpart relations that do not involve property-denoting indefinites.

\section{Conclusion}

We have seen ample motivation for analyzing $a$ indefinites as systematically ambiguous between properties and existential quantifiers. In locative PPs, this ambiguity allows us to capture the parallelism between the pseudo-quantificational behavior of $a$ indefinites and the similar behavior of referential NPs with a part-whole structure. We saw advantages to the property-based approach over a possible attempt to decompose the meaning of locatives by using negation. Further, we saw support for our approach coming from the behavior of NPIs and collective descriptions within locative PPs. This gives substantial support for the property analysis of indefinites within a new empirical domain: the spatial semantics of prepositional phrases.

The results reported in this paper also have some more general implications. The proposal that speakers can express pseudo-quantificational statements by virtue of the meaning of locative relations is only one instance of a recurrent theme in semantic theory: the idea that lexical meanings of predicates may lead to pseudo-quantificational effects. This idea goes back to Milsark and Carlson's treatments of indefinites in the 1970s. Similar views were expressed in Kroch (1974) and Scha (1981) in relation to the semantics of plurals and distributivity. The link between pseudo-quantification and lexical knowledge is central for semantic theory, an area that is caught between questions about syntactic structure and problems of mental concept modeling. Further, existential quantifiers in our account are not lexical denotations of certain indefinite articles. Rather, we view existential quantification as part of a derivational process in natural languages, possibly in all of them. When an indefinite denotes a property, existential mechanisms may or may not be at work, depending on the syntactic environment. This idea too goes back at least to Milsark and Carlson's works, but it is also prominent in theories based on Partee's flexibility thesis, as well as in treatments of event variables, unselective binding of pronouns, choice functions, and other influential works in formal semantics over the last 40 years. We believe that the present study may help to further extend this line of work into a comprehensive theory of indefiniteness, spatial expressions and part-whole structure.

Acknowledgments This paper develops our work in Mador-Haim and Winter (2007). We are greatly indebted to Heidi Klockmann, Louise McNally, and Joost Zwarts, as well as to Lisa Matthewson and two $L \& P$ reviewers for many remarks and suggestions. Thanks also to Sophie Chesney, Hanna de Vries, Martin Everaert, Danny Fox, Berit Gehrke, Robert Grimm, Riny Huybregts, Sabine Iatridou, Ed Keenan, Choonkyu Lee, Beth Levin, Anton Nguyen, Eva Poortman, Yael Seggev, Benjamin Spector, Eddy Ruys, Kai von Fintel and Ede Zimmermann, as well as to audiences at SALT17 (UConn), Stanford, Utrecht, MIT, UCLA, Frankfurt, Technion, Trento and Paris. The work of the second author was partially supported by a VICI Grant 277-80-002 of the Netherlands Organisation for Scientific Research (NWO). 
Open Access This article is distributed under the terms of the Creative Commons Attribution 4.0 International License (http://creativecommons.org/licenses/by/4.0/), which permits unrestricted use, distribution, and reproduction in any medium, provided you give appropriate credit to the original author(s) and the source, provide a link to the Creative Commons license, and indicate if changes were made.

\section{References}

Barker, C. (2015). Scope. In S. Lappin \& C. Fox (Eds.), Handbook of contemporary semantic theory (2nd ed.). Oxford: Wiley-Blackwell.

Barwise, J., \& Cooper, R. (1981). Generalized quantifiers and natural language. Linguistics and Philosophy, 4, 159-219.

Bianchi, V., \& Belletti, A. (2014). Indefinite subjects of unaccusatives. Lecture presented at the workshop specificity in the grammar: Form and interpretation, University of Trento, February 2014. Retrieved from http://clic.cimec.unitn.it/specificity/doc/bianchi-belletti-handout.pdf.

Büring, D. (2007). Cross-polar nomalies. In T. Friedman \& M. Gibson (Eds.), Proceedings of semantics and linguistic theory, SALT17 (pp. 37-52). Ithaca, NY: Cornell University.

Carlson, G. N. (1977). Reference to kinds in English. PhD Thesis, University of Massachusetts at Amherst.

Carlson, G. N., \& Pelletier, F. J. (1995). The generic book. Chicago: University of Chicago Press.

Casati, R., \& Varzi, A. C. (1999). Parts and places: The structures of spatial representation. Cambridge, MA: MIT Press.

Chierchia, G. (1998). Reference to kinds across languages. Natural Language Semantics, 6, 339-405.

Chung, S., \& Ladusaw, W. A. (2003). Restriction and saturation. Cambridge, MA: MIT Press.

Cohen, A. (2001). On the generic use of indefinite singulars. Journal of Semantics, 18, 183-209.

Condoravdi, C., Crouch, R., \& van den Berg, M. (2001). Counting concepts. In R. van Rooy \& M. Stokhof (Eds.), Proceedings of the thirteenth Amsterdam colloquium. Amsterdam: Institute for Logic, Language and Computation, University of Amsterdam.

Cruse, D. A. (1979). On the transitivity of the part-whole relation. Journal of Linguistics, 15, 29-38.

Dayal, V. (1999). Bare NP's, reference to kinds, and incorporation. In T. Matthews \& D. Strolovitch (Eds.), Proceedings of semantics and linguistic theory, SALT9 (pp. 34-51). Ithaca, NY: Cornell University.

de Hoop, H., \& Kramer, I. (2006). Optimal interpretations of indefinite subjects and objects. Language Acquisition, 13, 103-123.

Doron, E. (1983). Verbless predicates in Hebrew. PhD Thesis, The University of Texas at Austin.

Farkas, D. F., \& de Swart, H. (2003). The semantics of Incorporation: From argument structure to discourse transparency. Stanford, CA: CSLI Publications.

Fauconnier, G. (1975). Polarity and the scale principle. In R. E. Grossman, L. J. San, \& T. J. Vance (Eds.), Papers from the eleventh regional meeting of the Chicago Linguistic Society (pp. 188-199). Chicago: Chicago Linguistic Society.

Fodor, J. D., \& Sag, I. (1982). Referential and quantificational indefinites. Linguistics and Philosophy, 5, 355-398.

Giannakidou, A. (2001). The meaning of free choice. Linguistics and Philosophy, 24, 659-735.

Giannakidou, A. (2011). Negative and positive polarity items. In K. von Heusinger, C. Maienborn, \& P. Portner (Eds.), Semantics: An international handbook of natural language meaning (Vol. 2, pp. 1660-1712). Berlin: De Gruyter.

Greenberg, Y. (2007). Exceptions to generics: Where vagueness, context dependence and modality interact. Journal of Semantics, 24, 131-167.

Grimm, R. M., Lee, C., Poortman, E. B., \& Winter, Y. (2014). Evidence for non-existential readings of locative indefinites. In T. Snider, S. D’Antonio, \& M. Wiegand (Eds.), Proceedings of semantics and linguistic theory, SALT24 (pp. 197-212). Ithaca, NY: LSA and CLC Publications.

Groenendijk, J., \& Stokhof, M. (1991). Dynamic predicate logic. Linguistics and Philosophy, 14, 39-100.

Heim, I. (1982). The semantics of definite and indefinite noun phrases. PhD Thesis, University of Massachusetts at Amherst.

Heim, I. (2008). Decomposing antonyms? In A. Grønn (Ed.), Proceedings of SuB12 (pp. 212-225). Oslo: ILOS.

Herskovits, A. (1986). Language and spatial cognition: An interdisciplinary study of the prepositions in English. Cambridge: Cambridge University Press. 
Iatridou, S. (2003). Two constructions in Greek and what we can learn from them. In Proceedings of the 6th international conference of Greek linguistics (Vol. 6).

Iatridou, S. (2007). A free-choice item hidden in verbal morphology. Unpublished MS, MIT.

Iris, M. A., Litowitz, B. E., \& Evens, M. (1988). Problems of the part-whole relation. In M. Evens (Ed.), Relational models of the lexicon (pp. 261-288). Cambridge: Cambridge University Press.

Johansson, I. (2004). On the transitivity of parthood relations. In H. Hochberg \& K. Mulligan (Eds.), Relations and predicates (pp. 161-181). Frankfurt: Ontos/Lancaster.

Kamp, H. (1981). A theory of truth and semantic representation. In J. Groenendijk, M. Stokhof, \& T. M. V. Janssen (Eds.), Formal methods in the study of language (pp. 277-322). Amsterdam: Mathematisch Centrum.

Kamp, H., \& Reyle, U. (1993). From discourse to logic: Introduction to modeltheoretic semantics of natural language, formal logic and discourse representation theory. Dordrecht: Kluwer.

Keenan, E. L., \& Westerståhl, D. (2011). Generalized quantifiers in linguistics and logic. In J. van Benthem \& A. ter Meulen (Eds.), Handbook of logic and language (Vol. 2, pp. 859-910). Amsterdam: Elsevier.

Kelley, J. L. (1955). General topology. New York: Springer.

Kroch, A. S. (1974). The semantics of scope in English. PhD Thesis, Massachusetts Institute of Technology.

Ladusaw, W. A. (1979). Polarity sensitivity as inherent scope relations. PhD Thesis, University of Texas, Austin.

Landman, F. (2014). Chapter 9: Predicates and properties. Unpublished class notes, Tel-Aviv University. Retrieved from http://www.tau.ac.il/landman/online-class-notes.html.

Lang, S. (1977). Linear algebra (2nd ed.). Reading, MA: Addison-Wesley.

Lawler, J. M. (1973). Studies in English generics. PhD Thesis, University of Michigan.

Mador-Haim, S., \& Winter, Y. (2007). Non-existential indefinites and semantic incorporation of PP complements. In T. Friedman \& M. Gibson (Eds.), Proceedings of semantics and linguistic theory, SALT17 (pp. 184-201). Ithaca, NY: Cornell University.

McNally, L. (1998). Existential sentences without existential quantification. Linguistics and Philosophy, 21, 353-392.

McNally, L. (2009). Properties, entity correlates of properties, and existentials. In A. Giannakidou \& M. Rathert (Eds.), Quantification, definiteness, and nominalization (pp. 163-187). Oxford: Oxford University Press.

McNally, L. (2011). Existential sentences. In K. von Heusinger, C. Maienborn, \& P. Portner (Eds.), Semantics: An international handbook of natural language meaning (Vol. 2, pp. 1829-1848). Berlin: De Gruyter.

McNally, L. (2014). Strong and weak nominals. To appear in L. Matthewson, C. Meier, H. Rullmann, \& T. E. Zimmermann (Eds.), Blackwell companion to semantics. New York: Wiley-Blackwell.

Milsark, G. (1974). Existential sentences in English. PhD Thesis, Massachusetts Institute of Technology.

Moltmann, F. (1997). Parts and wholes in semantics. New York: Oxford University Press.

Montague, R. (1973). The proper treatment of quantification in ordinary English. In J. Hintikka, J. Moravcsik, \& P. Suppes (Eds.), Approaches to natural languages: Proceedings of the 1970 Stanford workshop on grammar and semantics. Dordrecht: D. Reidel. Reprinted from Formal philosophy: Selected papers of Richard Montague, by R. Thomason, Ed., 1974, New Haven: Yale.

Nam, S. (1994). Another type of negative polaity item. In M. Kanazawa \& C. J. Piñón (Eds.), Dynamics, polarity and quantification (pp. 3-15). Stanford, CA: CSLI Publications.

Partee, B. (1987). Noun phrase interpretation and type shifting principles. In J. Groenendijk, D. de Jong, $\&$ M. Stokhof (Eds.), Studies in discourse representation theories and the theory of generalized quantifiers (pp. 115-144). Dordrecht: Foris.

Reinhart, T. (1997). Quantifier scope: How labor is divided between QR and choice functions. Linguistics and Philosophy, 20, 335-397.

Scha, R. (1981). Distributive, collective and cumulative quantification. In J. Groenendijk, M. Stokhof, \& T. M. V. Janssen (Eds.), Formal methods in the study of language (pp. 483-512). Amsterdam: Mathematisch Centrum.

Szabolcsi, A. (2004). Positive polarity-negative polarity. Natural Language and Linguistic Theory, 22, 409-452.

Thijsse, E. (1983). On some proposed universals of natural language. In A. ter Meulen (Ed.), Studies in model theoretic semantics (pp. 19-36). Dordrecht: Foris.

Van Geenhoven, V. (1996). Semantic incorporation and indefinite descriptions: Semantic and syntactic aspects of noun incorporation in West Greenlandic. PhD Thesis, Universität Tübingen. 
Van Geenhoven, V. (1998). Semantic incorporation and indefinite descriptions: Semantic and syntactic aspects of noun incorporation in West Greenlandic. Stanford, CA: CSLI Publications.

Van Geenhoven, V., \& McNally, L. (2005). On the property analysis of opaque complements. Lingua, 115, 885-914.

von Fintel, K. (1999). NPI licensing, Strawson entailment, and context dependency. Journal of Semantics, 16, 97-148.

von Fintel, K. (2004). Would you believe it? The king of France is back! (presuppositions and truth-value intuitions). In M. Reimer \& A. Bezuidenhout (Eds.), Descriptions and beyond (pp. 315-341). Oxford: Oxford University Press.

Von Heusinger, K. (2011). Specificity. In K. von Heusinger, C. Maienborn, \& P. Portner (Eds.), Semantics: An international handbook of natural language meaning (Vol. 2, pp. 1025-1057). Berlin: De Gruyter.

Winston, M., Chaffin, R., \& Herrmann, D. (1987). A taxonomy of part-whole relations. Cognitive Science, $11,417-444$.

Winter, Y. (2001). Flexibility principles in Boolean semantics: Coordination, plurality and scope in natural language. Cambridge, MA: MIT Press.

Winter, Y., \& Scha, R. (2015). Plurals. In S. Lappin \& C. Fox (Eds.), Handbook of contemporary semantic theory (Vol. 2). Oxford: Wiley-Blackwell.

Wunderlich, D. (1991). How do prepositional phrases fit into compositional syntax and semantics? Linguistics, 29, 591-621.

Zimmermann, T. E. (1993). On the proper treatment of opacity in certain verbs. Natural Language Semantics, $1,149-179$.

Zwarts, F. (1998). Three types of polarity. In F. Hamm \& E. Hinrichs (Eds.), Plurality and quantification (pp. 177-238). Dordrecht: Kluwer.

Zwarts, J., \& Winter, Y. (2000). Vector space semantics: A model-theoretic analysis of locative prepositions. Journal of Logic, Language and Information, 9, 169-211. 